\title{
Distributed Resource Allocation Assisted by Intercell Interference Mitigation in Downlink Multicell MC DS-CDMA Systems
}

\author{
Jia Shi, Zhengyu Song, IEEE Member, and Qiang Ni, IEEE Senior Member
}

\begin{abstract}
This paper investigates the allocation of resources including subcarriers and spreading codes, as well as intercell interference (ICI) mitigation for multicell downlink multicarrier direct-sequence code division multiple-access (MC DS-CDMA) systems, which aim to maximize the system's spectral efficiency (SE). The analytical benchmark scheme for resource allocation and ICI mitigation is derived by solving or closely solving a series of mixed integer non-convex optimization problems. Based on the optimization objectives same as the benchmark scheme, we propose a novel distributed resource allocation assisted by ICI mitigation scheme referred to as RAIM, which requires very low implementation complexity and demands little backhaul resource. Our RAIM algorithm is a fully distributed algorithm, which consists of the subcarrier allocation (SA) algorithm named RAIM-SA, spreading code allocation (CA) algorithm called RAIM-CA and the ICI mitigation algorithm termed RAIM-IM. The advantages of the RAIM include that, its CA only requires limited binary ICI information of intracell channels, and it is able to make mitigation decisions without any knowledge of ICI information. Our simulation results show that, the proposed RAIM scheme with very low complexity required achieves significantly better $\mathrm{SE}$ performance than other existing schemes, and its performance is very close to that obtained by the benchmark scheme.
\end{abstract}

Index Terms-Resource allocation, multicell, MC DS-CDMA, intercell interference mitigation.

\section{INTRODUCTION}

Dynamic resource allocation has become more and more important in future broadband multicarrier communications by exploiting time-varying characteristics of wireless channels and making use of multiuser diversity. However, resource allocation also faces a lot of challenges, including the possible huge signaling overhead, limited backhaul resources, high-complexity backhaul operations, expansive servicing area, highly diverse services, etc. Resource allocation in single-cell multicarrier systems has been widely investigated in [1-4]. However, it becomes very challenging in multicell systems due to severe intercell interference (ICI) caused by frequency reuse.

Resource allocation in multicell multicarrier systems can be categorized into two classes, namely centralized and distributed approaches. Specifically, centralized resource allocation has been widely investigated and studied in multicell scenarios, such as $[5,6]$. Despite exploiting various degrees of freedom

This work is supported by EU H2020 TWEETHER project under grant agreement number 644678 .

J. Shi and Q. Ni are with School of Computing and Communications, Lancaster University, LA1 4WA, UK. Z. Song is with the School of Electronic and Information Engineering, Beijing Jiaotong University, Beijing 100044, China. (E-mail: j.shi@lancaster.ac.uk, z.song912@gmail.com, q.ni@lancaster.ac.uk). in terms of allocation, centralized approach usually demands a very high implementation complexity and backhaul resources. By contrast, when distributed approach is used, resources can be managed and assigned independently by each base station (BS). In comparison with centralized approach, distributed resource allocation requires a significantly lower complexity, and it can also release huge burden on the backhaul system and reduce massive signaling overhead. Furthermore, distributed approach is able to quickly respond to the dynamic and fast varying channel environments of wireless communication systems. Exploiting the above advantages, distributed resource allocation will be more and more useful and desirable in future wireless systems considering a large number of cells. Hence, distributed resource allocation has attracted intensive attentions, e.g. [7-10] in multicell orthogonal frequency division multiple access (OFDMA) systems. However, some distributed schemes, such as those in $[8,9]$, still need BSs to exchange channel state information (CSI) of their users, as a result, these schemes are less competitive for practical implementation. On the other hand, due to lack of ICI information, combating ICI for distributed resource allocation is more challenging than that for centralized resource allocation. In the literature, BS cooperation is an efficient ICI mitigation approach, which shifts the processing burden from mobile terminals to BSs. The authors in $[11,12]$ have studied the scheduling and power-allocation in the multicell downlink OFDMA networks, which handles ICI via $\mathrm{BS}$ coordination supported by CSI exchange among BSs. By contrast, the researches in $[13,14]$ have studied the resource allocation in the multicell systems with full BS cooperation requiring both CSI and data information exchanged among BSs, which is however not allowed in many practical scenarios.

In comparison with the other multicarrier schemes without using direct-sequence (DS) spreading, such as OFDMA and MC-CDMA, multicarrier DS code division multiple-access (MC DS-CDMA) employs a high number of degrees-offreedom for high-flexibility design and reconfiguration [15]. With the aid of DS spreading, MC DS-CDMA is able to employ a significant lower number of subcarriers than OFDMA [16], and, hence, can mitigate the peak to average power ratio (PAPR) problem. Wireless signals in multicarrier schemes may experience severe frequency-selective fading, which significantly degrades system performance if it is not properly handled. MC DS-CDMA employs the flexibility to configure its number of subcarriers according to the frequency-selectivity of wireless channels. As a result, each subcarrier experiences independent fading, and frequency diversity may be attained by conveying 
the same information using different subcarriers, which are then coherently combined at receiver. In this case, the number of subcarriers of MC DS-CDMA will be at the order of the number of time domain resolvable paths of wireless channels and, hence, will usually be low [16]. By exploiting the abovementioned advantages, MC DS-CDMA can be a promising solution for supporting ubiquitous wireless communications in diverse environments, such as indoor, rural, and urban areas. It is feasible for accessing a large and possibly discontinuous bandwidth. This property is especially beneficial to the cognitive radio systems, where the bandwidth for supporting multiuser communications is obtained from the spectrum holes of primary radio systems. However, very limited researches, such as [17-22], have been devoted to resource allocation in MC DS-CDMA systems. In $[17,18]$, the joint allocation of subcarrier and non-orthogonal spreading codes have been studied in the single-cell MC DS-CDMA systems. Moreover, a code assignment scheme with multiple-access interference avoidance has been proposed for the generalized MC DS-CDMA systems in $[19,20]$. Recently, with the aid of non-cooperative game approach, the paper in [21] has addressed the resource allocation including only transmit power and subchannels for the multicell distributed MC DS-CDMA network. The authors in [22] have proposed a joint spreading code allocation and subcarrier scrambling scheme to reduce the PAPR in the singlecell MC CDMA system, where the interference issue has not been addressed. In our paper [23], we have investigated lowcomplexity subcarrier allocation in the single-cell MC DSCDMA systems. However, efficient resource allocation in multicell scenarios will be very challenging due to existence of strong ICI as well as high implementation complexity.

To the best of our knowledge, there are no published references that have studied resource allocation and interference mitigation together for the multicell MC DS-CDMA systems. Against the background, in this paper, we investigate how to efficiently allocate resources including subcarriers and spreading codes, while effectively mitigating ICI for multicell downlink MC DS-CDMA systems, which aims to maximize the system's spectral efficiency (SE). The main contributions of our paper can be summarized as follows.

- We derive a novel benchmark scheme for the distributed resource allocation and ICI mitigation in the multicell MC DS-CDMA systems. The benchmark scheme proposes an efficient approach to allocate subcarriers and codes, while mitigating ICI, which can be obtained by solving and closely solving a series of mixed integer non-convex optimization problems. As proposed, the distributed subcarrier allocation (SA) is first carried out, then the distributed spreading code allocation (CA) is implemented, aiming to maximize the sum rate of each cell. After that, the ICI mitigation is operated for maximizing the sum rate of celledge users.

- Inspired by the benchmark scheme, we propose a novel low-complexity scheme, namely distributed resource allocation assisted by ICI mitigation (RAIM), for the multicell downlink MC DS-CDMA systems. Based on the optimization objectives same as the benchmark scheme, our RAIM scheme is designed to be of very low implementation complexity and small backhaul resource demanded. The RAIM first operates the distributed SA algorithm named RAIM-SA, then runs the CA algorithm called RAIMCA. In contrast to the benchmark, after the SA and CA the RAIM carries out the fully distributed ICI mitigation algorithm termed RAIM-IM.

- We analyze the characteristics and complexity of the RAIM and the benchmark schemes employed by our multicell MC DS-CDMA systems. The proposed schemes have a range of advantages, including quick response to wireless channel environments, requring minimum backhaul burden and minimum load on feedback channels, as well as easily applicable for large system, etc. According to the complexity analysis, the RAIM scheme requires much lower complexity than the benchmark scheme, which implies the RAIM is very advantegeous in practical implementation.

- We carry out comprehensive performance analysis for the proposed RAIM and benchmark schemes employed by the multicell systems. It is shown that, the proposed RAIM scheme achieves significantly better SE performance than other existing distributed resource allocation algorithms, and its performance is very close to that obtained by the benchmark scheme. Therefore, the MC DS-CDMA associated with the RAIM scheme may constitute a promising candidate that facilitates practical implementation in future communication systems.

The rest of this paper is organized as follows. Section II introduces the system model, and states our optimization problems. Section III derives the benchmark for our resource allocation. Section IV proposes the novel RAIM scheme. Section $\mathrm{V}$ analyzes the characteristics and complexity of the proposed schemes. Performance results are shown in Section VI. Finally, we summarize the main conclusions in Section VII.

\section{System Model AND Problem Formulation}

In this section, we present the system model followed by describing the main optimization problems for the resource allocation and ICI mitigation in the multicell MC DS-CDMA systems.

\section{A. System Model}

To reflect the main features of multicell systems, we consider the classical three-cell system model, which has been widely studied in $[14,24,25]$. As shown in Fig. 1, a BS locates at the center of a hexagonal cell, and its $K$ users uniformly distribute in the cell. Each of the communication terminals is equipped with one antenna for signal transmission and reception. Furthermore, signals transmitted from BSs to mobile users are MC DSCDMA signals employing length- $N$ orthogonal DS spreading codes (or, simply, codes) and in total $M$ orthogonal subcarriers. We assume that each cell supports $K=M N$ users and, hence, each user can be allocated one subcarrier and one code. Note that, we consider this extreme case for both the SA and CA in the systems, as it is the most challenging one while avoiding considering different trivial cases. 


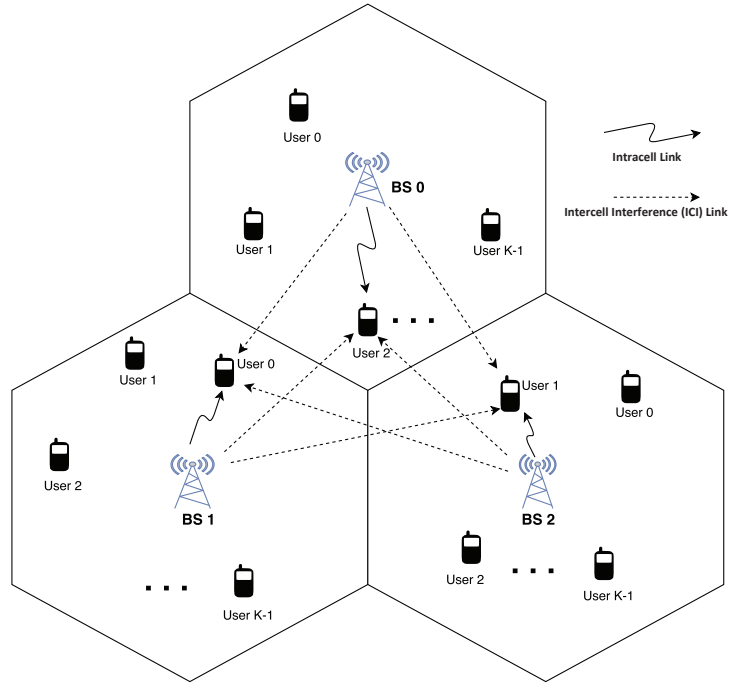

Fig. 1: Conceptual structure of the downlink multicell MC DSCDMA systems.

In order to avoid intracell interference, users in the same cell are allocated either different subcarriers or different codes, or both are different. However, the "co-subcarrier-code" users in different cells assigned the same subcarrier and code, such as users 2, 0 and 1 of cells 0,1 and 2 in Fig. 1, will cause ICI to each other. Furthermore, in each cell we assume for simplicity ideal power control as in $[1,13,26]$, for maintaining the same average received power of one unit per user when there are no SA and CA. In that case, we define an ICI factor $\alpha$, considering the combined effects of propagation pathloss and shadowing, as [27]

$$
\alpha=\sqrt{\left(\frac{d_{0}}{d_{1}}\right)^{\mu} 10^{\frac{\zeta_{0}-\zeta_{1}}{10}}}
$$

where $d_{0}$ and $d_{1}$ represent the distances from a BS to its intracell and intercell users respectively. In (1), $\mu$ is the pathloss exponent, and $\left(\zeta_{0}-\zeta_{1}\right)$ (in $\left.\mathrm{dB}\right)$ obeys the log-normal distribution with standard deviation $\Upsilon$ (in $\mathrm{dB}$ ), which accounts for the shadowing effect [27]. In addition, each transmission also experiences fast fading, which is assumed to be the independent Rayleigh flat fading.

Let us assume that the data symbols transmitted by BS $u(u \in$ $\mathcal{U}=\{0,1,2\})$ to its $K$ intracell users are expressed as $\boldsymbol{x}^{(u)}=$ $\left[x_{0}^{(u)}, x_{1}^{(u)}, \ldots, x_{K-1}^{(u)}\right]^{T}$, where $x_{k}^{(u)}$ is the data symbol to user $k$, and is assumed to satisfy $E\left[x_{k}^{(u)}\right]=0$ and $E\left[\left|x_{k}^{(u)}\right|^{2}\right]=1$. Assume that users $k, k^{\prime}$ in cells $u, u^{\prime}$ are co-subcarrier-code users, which are assigned subcarrier $m$ and code $n$. The signals received by user $k$ can be written by

$$
\begin{aligned}
\boldsymbol{y}_{k, m}^{(u)}= & h_{k, m}^{(u)} \boldsymbol{V}_{m} \boldsymbol{W}^{(u)} \boldsymbol{x}^{(u)}+\sum_{u^{\prime} \in \mathcal{U}, u^{\prime} \neq u} h_{k, m}^{\left(u^{\prime}\right)} \alpha_{k, k^{\prime}}^{\left(u^{\prime}\right)} \boldsymbol{V}_{m} \boldsymbol{W}^{\left(u^{\prime}\right)} \boldsymbol{x}^{\left(u^{\prime}\right)} \\
& +\boldsymbol{n}_{k}^{(u)}, \quad k \in \mathcal{K}^{(u)}, m \in \mathcal{M}
\end{aligned}
$$

where $\mathcal{K}^{(u)}$ contains the indexes of users in cell $u$, and $\mathcal{M}$ includes the indexes of subcarriers of the system. In (2), $\boldsymbol{y}_{k, m}^{(u)}$ is a length- $N$ observation vector, and $h_{k, m}^{(u)}$ is the fast fading channel gain from BS $u$ to user $k$ of subcarrier $m$. As defined in (1), $\alpha_{k, k^{\prime}}^{\left(u^{\prime}\right)}$ characterizes the ICI from BS $u^{\prime}$ to user $k$. We assume $V$ is a $(N \times K)$-dimensional matrix with columns consisting of the spreading sequences taken from a $(N \times N)$ orthogonal spreading code matrix. Hence, $V_{m}$ is a $(N \times K)$ dimensional matrix formed from $V$ by setting those columns for the subcarriers other than $m$ to zero vectors. The vector $\boldsymbol{n}_{k}^{(u)}=$ $\left[n_{k, 0}^{(u)}, \ldots, n_{k, N-1}^{(u)}\right]^{T}$ is a complex Gaussian noise vector at user $k$, which has zero mean and a variance of $2 \sigma^{2}=1 / \gamma_{s}$. We define $\gamma_{s}$ as the average signal-to-noise ratio (SNR) per symbol, when there is no SA/CA. We assume that a BS is capable of acquiring the CSI of its intracell channels. Hence, the preprocessing matrix can be set as $\boldsymbol{W}^{(u)}=\operatorname{diag}\left\{w_{0}^{(u)}, w_{1}^{(u)}, \ldots, w_{K-1}^{(u)}\right\}$ with $w_{k}^{(u)}=\left(h_{k, m}^{(u)}\right)^{*} / \sqrt{\left|h_{k, m}^{(u)}\right|^{2}}$, where $(\cdot)^{*}$ denotes the conjugate operation. However, to minimize backhaul burden and implementation complexity, BSs are not allowed to exchange the information about both intracell and ICI channels of the users. After despreading, the decision variable of user $k$ becomes

$$
\begin{aligned}
z_{k, m}^{(u)}= & \sqrt{\left|h_{k, m}^{(u)}\right|^{2}} x_{k}^{(u)}+\underbrace{\sum_{u^{\prime} \in \mathcal{U}, u^{\prime} \neq u} h_{k, m}^{\left(u^{\prime}\right)} \alpha_{k, k^{\prime}}^{\left(u^{\prime}\right)} w_{k^{\prime}}^{\left(u^{\prime}\right)} x_{k^{\prime}}^{\left(u^{\prime}\right)}}_{\text {ICI }} \\
& +n_{k}^{(u)}, \quad k \in \mathcal{K}^{(u)}, m \in \mathcal{M}
\end{aligned}
$$

From (3), we know the signal-to-interference-plus-noise ratio (SINR) of user $k$, given by

$$
\begin{aligned}
\gamma_{k, m}^{(u)} & =\frac{\left|h_{k, m}^{(u)}\right|^{2}}{\sum_{u^{\prime} \in \mathcal{U}, u^{\prime} \neq u}\left|h_{k, m}^{\left(u^{\prime}\right)} \alpha_{k, k^{\prime}}^{\left(u^{\prime}\right)} w_{k^{\prime}}^{\left(u^{\prime}\right)}\right|^{2}+2 \sigma^{2}} \\
& =\frac{1}{\left(A_{k, m}^{(u)}\right)^{-1}+\left(\eta_{k, m}^{(u)}\right)^{-1}}, k \in \mathcal{K}^{(u)}, m \in \mathcal{M}
\end{aligned}
$$

where $A_{k, m}^{(u)}$ and $\eta_{k, m}^{(u)}$ are respectively the SNR and signalto-interference (SIR) for user $k$ of subcarrier $m$. They can be written as

$$
\begin{gathered}
A_{k, m}^{(u)}=\frac{\left|h_{k, m}^{(u)}\right|^{2}}{2 \sigma^{2}}=\gamma_{s}\left|h_{k, m}^{(u)}\right|^{2}, \eta_{k, m}^{(u)}=\frac{\left|h_{k, m}^{(u)}\right|^{2}}{\sum_{u^{\prime} \in \mathcal{U}, u^{\prime} \neq u} I_{k, k^{\prime}, m}^{\left(u^{\prime}\right)}}, \\
k \in \mathcal{K}^{(u)}, k^{\prime} \in \mathcal{K}^{\left(u^{\prime}\right)}, m \in \mathcal{M}
\end{gathered}
$$

where $I_{k, k^{\prime}, m}^{\left(u^{\prime}\right)}=\left|h_{k, m}^{\left(u^{\prime}\right)} \alpha_{k, k^{\prime}}^{\left(u^{\prime}\right)} w_{k^{\prime}}^{\left(u^{\prime}\right)}\right|^{2}$ is the ICI power of user $k$ received from $\mathrm{BS} u^{\prime}$.

\section{B. Problem Formulation}

This section discusses the general theories and formulates the optimization problems for the distributed resource allocation and ICI mitigation in the multicell systems. First, the distributed resource allocation including $\mathrm{SA}$ and $\mathrm{CA}$ aims to maximize the sum rate of each cell, described as

$$
\left\{\mathcal{S}^{(u)}, \mathcal{C}^{(u)}\right\}^{*}=\arg \max _{\left\{\mathcal{S}^{(u)}, \mathcal{C}^{(u)}\right\}}\left\{\sum_{k \in \mathcal{K}^{(u)}} R_{k}^{(u)}\right\}, \forall u \in \mathcal{U}
$$




$$
R_{k}^{(u)}=\log _{2}\left(1+\frac{\sum_{m \in \mathcal{M}} s_{k, m}^{(u)}\left|h_{k, m}^{(u)}\right|^{2}}{\sum_{u^{\prime} \in \mathcal{U}, u^{\prime} \neq u} \sum_{k^{\prime} \in \mathcal{K}^{\left(u^{\prime}\right)}} \sum_{n \in \mathcal{N}} \sum_{m \in \mathcal{M}} c_{k, n}^{(u)} c_{k^{\prime}, n}^{\left(u^{\prime}\right)} s_{k, m}^{(u)} s_{k^{\prime}, m}^{\left(u^{\prime}\right)} I_{k, k^{\prime}, m}^{\left(u^{\prime}\right)}+2 \sigma^{2}}\right)
$$

subject to

$$
\begin{aligned}
& s_{k, m}^{(u)}=\{0,1\}, \forall m \in \mathcal{M}, \forall k \in \mathcal{K}^{(u)}, \\
& \sum_{k \in \mathcal{K}^{(u)}} s_{k, m}^{(u)}=N, \quad \forall m \in \mathcal{M}, \\
& \sum_{m \in \mathcal{M}} s_{k, m}^{(u)}=1, \quad \forall k \in \mathcal{K}^{(u)}, \\
& c_{k, n}^{(u)}=\{0,1\}, \quad \forall k \in \mathcal{K}^{(u)}, \forall n \in \mathcal{N}, \\
& \sum_{n \in \mathcal{N}} c_{k, n}^{(u)}=1, \quad \forall k \in \mathcal{K}^{(u)}, \\
& \sum_{k \in \mathcal{K}^{(u)}} c_{k, n}^{(u)}=M, \quad \forall n \in \mathcal{N} .
\end{aligned}
$$

Above, $R_{k}^{(u)}$, given by (7), is the achievable rate of user $k$ in cell $u$. In (6), $\mathcal{S}^{(u)}=\left\{s_{k, m}^{(u)}, \forall k, m\right\}$ and $\mathcal{C}^{(u)}=\left\{c_{k, n}^{(u)}, \forall k, n\right\}$ contain the SA and CA variables, while $\left\{\mathcal{S}^{(u)}, \mathcal{C}^{(u)}\right\}^{*}$ denotes the final results of cell $u$. The indicator $s_{k, m}^{(u)}=1$ if subcarrier $m$ is allocated to user $k$ in cell $u$, otherwise $s_{k, m}^{(u)}=0$. Similarly, the indicator $c_{k, n}^{(u)}=1$ if code $n$ is assigned to user $k$ in cell $u$, otherwise $c_{k, n}^{(u)}=0$. In above equations, $\mathcal{N}$ contains the indexes of codes in the system. Note that, the constraints of (9) and (10) follow the assumption that each subcarrier is assigned to $N$ users in one cell, while each user is allocated one subcarrier. By contrast, (12) and (13) constrain that each user is only allocated one code, and each code is assigned to $M$ users.

To solve problem (6), the BSs are required to share the full ICI information of all users, which is however not allowed by our assumption for achieving minimum backhaul burden and low implementation complexity. Therefore, limited by this assumption, each BS first independently carries out the SA for solving problem (14), then operates CA for solving problem (16) which can approximate problem (6). After that, ICI mitigation is implemented based on the optimization problem of (19), which aims to maximize the sum rate of the cell-edge users for further improving the system throughput. First, the distributed SA aims to maximize the sum rate of each cell, described as

$$
\left\{\mathcal{S}_{m}^{(u)}, \forall m\right\}^{*}=\arg \max _{\left\{\mathcal{S}_{m}^{(u)}, \forall m\right\}}\left\{\sum_{k \in \mathcal{K}^{(u)}} R_{k}^{(u)}\right\}, \forall u \in \mathcal{U}
$$

subject to (8), (9), and (10).

In (14), the achievable rate of user $k$, i.e. $R_{k}^{(u)}$, becomes

$$
R_{k}^{(u)}=\log _{2}\left(1+\frac{\sum_{m \in \mathcal{M}} s_{k, m}^{(u)}\left|h_{k, m}^{(u)}\right|^{2}}{\sum^{I_{k}}+2 \sigma^{2}}\right) .
$$

In (14), $\mathcal{S}_{m}^{(u)}=\left\{s_{k, m}^{(u)}, \forall k\right\}$ contains the SA variables of cell $u$. Note that in (15), $\sum I_{k}$ denotes the sum of the ICI experienced by user $k$, and it can be given by the first term of the SINR's denominator in (7). However, we focus on studying distributed resource allocation, and the BSs are not allowed to share the full ICI information of all users and, hence, $\sum_{\sum} I_{k}$ in (15) cannot be known. Furthermore, each user's ICI only comes from its co-subcarrier-code users, which can be known after the SA and CA. Hence, users' ICI cannot be determined when carrying out the SA based on (14).

After SA, each BS independently carries out CA, which aims to maximize the sum rate of each cell. In order to avoid intracell interference, the co-subcarrier users in a cell are distinguished by different codes. Therefore, based on the SA results, maximizing the sum rate of a cell is equivalent to independently maximizing the sum rate of every co-subcarrier user group in a cell. Correspondingly, the CA problem can be expressed as

$$
\begin{aligned}
\left\{\mathcal{C}_{m}^{(u)}\right\}^{*}= & \arg \max _{\left\{\mathcal{C}_{m}^{(u)}\right\}}\left\{\sum_{k \in \mathcal{F}_{m}^{(u)}} R_{k}^{(u)} \mid\left\{\mathcal{S}_{m}^{(u)}\right\}^{*}\right\}, \\
& \forall m \in \mathcal{M}, \forall u \in \mathcal{U}
\end{aligned}
$$

subject to (11), (12), and

$$
\sum_{k \in \mathcal{F}_{m}^{(u)}} c_{k, n}^{(u)}=1, \forall n \in \mathcal{N} .
$$

In (16), $\mathcal{C}_{m}^{(u)}=\left\{c_{k, n}^{(u)}, \forall k \in \mathcal{F}_{m}^{(u)}, \forall n\right\}$ contains the CA variables for the users allocated subcarrier $m$, where $\mathcal{F}_{m}^{(u)}$ includes the indexes of the users assigned to subcarrier $m$ in cell $u$. For avoiding intracell interference, (17) constrains the users in a co-subcarrier user group are assigned different codes. In (16), the rate of user $k$ can be expressed by

$R_{k}^{(u)}=\log _{2}\left(1+\frac{\left|h_{k, m}^{(u)}\right|^{2}}{\sum_{u^{\prime} \in \mathcal{U}, u^{\prime} \neq u} \sum_{k^{\prime} \in \mathcal{F}_{m}^{\left(u^{\prime}\right)}} \sum_{n \in \mathcal{N}} c_{k, n}^{(u)} c_{k^{\prime}, n}^{\left(u^{\prime}\right)} I_{k, k^{\prime}, m}^{\left(u^{\prime}\right)}+2 \sigma^{2}}\right)$.

Note that, (18) is derived by removing all the SA variables in (7), since SA has been done. In addition, seen from (18), each user's potential ICI can only come from its co-subcarrier users in the other cells.

Finally, based on the SA and CA, our ICI mitigation is attempted for cell-edge users suffering from strong ICI. Let us define $\hat{\mathcal{K}}^{(u)}=\left\{k \mid \eta_{k}<\eta_{t}, k \in \mathcal{K}^{(u)}\right\}$ as the set of users in cell $u$, whose SIRs are below the SIR threshold $\eta_{t}$, which can be set according to various communication objectives. Then, cell-edge users are collected into $\tilde{\mathcal{K}}^{(u)}$, which includes both the users in $\hat{\mathcal{K}}^{(u)}$ as well as the users in $\left(\mathcal{K}^{(u)}-\hat{\mathcal{K}}^{(u)}\right)$ that share the same subcarriers and codes as the users in $\hat{\mathcal{K}}^{\left(u^{\prime}\right)}$ and $\hat{\mathcal{K}}^{\left(u^{\prime \prime}\right)}$. Therefore, the ICI mitigation for cell-edge users aims to maximize the sum rate of every cell-edge user group containing three co-subcarrier-code users. The optimization problem can be expressed as

$$
\begin{aligned}
\left\{\mathcal{D}_{m, n}\right\}^{*} & =\arg \max _{\left\{\mathcal{D}_{m, n}\right\}}\left\{\sum_{u \in \mathcal{U}} \sum_{k \in \mathcal{B}_{m, n}^{(u)}} R_{k}^{(u)} \mid\{\mathcal{S}, \mathcal{C}\}^{*}\right\}, \\
& \forall m \in \mathcal{M}, \forall n \in \mathcal{N}
\end{aligned}
$$


subject to

$$
\begin{aligned}
& d_{m, n}^{(u)}=\left\{k, k^{\prime}, k^{\prime \prime},-1\right\}, \\
& \text { if } k \in \mathcal{B}_{m, n}^{(u)} \& k^{\prime} \in \mathcal{B}_{m, n}^{\left(u^{\prime}\right)} \& k^{\prime \prime} \in \mathcal{B}_{m, n}^{\left(u^{\prime \prime}\right)}, \forall u \in \mathcal{U}, \\
& \sum_{u=0}^{U-1} d_{m, n}^{(u)} \geq-2, \\
& d_{m, n}^{(u)} \neq d_{m, n}^{\left(u^{\prime \prime}\right)}, \text { if } d_{m, n}^{(u)}=d_{m, n}^{\left(u^{\prime}\right)}=k, \quad \forall u .
\end{aligned}
$$

Above, $\mathcal{B}_{m, n}^{(u)}=\tilde{\mathcal{K}}^{(u)} \cap \mathcal{F}_{m}^{(u)} \cap \mathcal{V}_{n}^{(u)}$ is the set of indexes of the cell-edge users assigned subcarrier $m$ and code $n$, in which $\mathcal{V}_{n}^{(u)}$ contains the indexes of the users assigned to code $n$ in cell $u$. Similarly, $\mathcal{B}_{m, n}^{\left(u^{\prime}\right)}=\tilde{\mathcal{K}}^{\left(u^{\prime}\right)} \cap \mathcal{F}_{m}^{\left(u^{\prime}\right)} \cap \mathcal{V}_{n}^{\left(u^{\prime}\right)}$ and $\mathcal{B}_{m, n}^{\left(u^{\prime \prime}\right)}=\tilde{\mathcal{K}}^{\left(u^{\prime \prime}\right)} \cap$ $\mathcal{F}_{m}^{\left(u^{\prime \prime}\right)} \cap \mathcal{V}_{n}^{\left(u^{\prime \prime}\right)}$. Further, we define ICI mitigation decision (IMD) variable set as $\mathcal{D}_{m, n}=\left\{d_{m, n}^{(u)}, \forall u \in \mathcal{U}\right\}$ for subcarrier $m$ and code $n$. As described by (20), variable $d_{m, n}^{(u)}$ can be defined as

$$
\begin{gathered}
d_{m, n}^{(u)}= \begin{cases}k & \begin{array}{l}
\text { BS } u \text { transmits } x_{k}^{(u)} \text { to user } k \text { on subcarrier } m \\
\text { and code } n,
\end{array} \\
-1 & \begin{array}{l}
\text { BS } u \text { turns off its transmission on subcarrier } m \\
\text { and code } n,
\end{array} \\
k^{\prime}\left(\text { or } k^{\prime \prime}\right) & \begin{array}{l}
\text { BS } u \text { helps to send } x_{k^{\prime}}^{\left(u^{\prime}\right)}\left(\text { or } x_{k^{\prime \prime}}^{\left(u^{\prime \prime}\right)}\right) \text { to user } k^{\prime} \\
\left(\text { or } k^{\prime \prime}\right) \text { on subcarrier } m \text { and code } n,
\end{array} \\
\text { if } k \in \mathcal{B}_{m, n}^{(u)}, k^{\prime} \in \mathcal{B}_{m, n}^{\left(u^{\prime}\right)}, k^{\prime \prime} \in \mathcal{B}_{m, n}^{\left(u^{\prime \prime}\right)} .\end{cases}
\end{gathered}
$$

As shown by (23), two strategies, i.e. power off and cooperation, are jointly utilized for ICI mitigation. The system's SE may be significantly improved by switching off some transmissions which impose strong ICI on other transmissions [28]. Furthermore, when cooperation is available between two BSs, the space time block coding (STBC) [29] aided BS cooperation can be a promising scheme, which only needs to exchange data symbols, but no CSI, between the two BSs. (21) and (22) constrains that at most two users are allowed to switch off, and only two BSs can cooperate for a user in a cell-edge group.

So far, we can readily know that the optimization problems in (14), (16) and (19) for the SA, CA and the ICI mitigation are mixed integer non-convex problems, which are extremely hard to solve. In Section III, we motivate to solve the problems in (14), (16) and (19) respectively, resulting in a benchmark scheme. Furthermore, aiming to obtaining promising sub-optimum solutions, we propose a novel low-complexity scheme, namely RAIM, which follows the design objectives of the benchmark scheme.

\section{Benchmark AnAlysis of Resource Allocation AND ICI MITIGATION}

In this section, we derive a novel benchmark scheme for the resource allocation and ICI mitigation in multicell downlink MC DS-CDMA systems. The benchmark scheme is obtained by closely solving the problems in (14) and (16), as well as solving problem (19). The benchmark scheme consists of the distributed SA algorithm referred to as the benchmark-SA, the distributed CA algorithm termed as benchmark-CA and the centralized ICI mitigation algorithm called as benchmark-IM. In order to solve the problem of (19), the BSs are only allowed to exchange the ICI information of cell-edge users.

\section{A. Benchmark of Distributed Subcarrier Allocation: Benchmark-SA}

Our distributed SA aims to maximize the sum rate of the users in each cell, as shown in (14). However, to solve (14) it requires all users' ICI information exchanged among the BSs, which is not allowed in our assumption and in most of practical systems. In this case, as done in $[23,30]$ and the references therein, the best sub-optimum solution to problem (14) can be achieved by maximizing every user's SNR without considering ICI effect, having the distributed SA problem of

$$
\begin{aligned}
& \left\{\mathcal{S}_{m}^{(u)}, \forall m\right\}^{*}= \\
& \arg \max _{\left\{\mathcal{S}_{m}^{(u)}, \forall m\right\}}\left\{\sum_{m \in \mathcal{M}} s_{k, m}^{(u)} A_{k, m}^{(u)}, \forall k \in \mathcal{K}^{(u)}\right\}, \forall u \in \mathcal{U}
\end{aligned}
$$

subject to (8), (9) and (10).

However, the problem of (24) is still a combinatorial problem, which is very hard to solve. By contrast, in $[2,31]$, the authors have proved that the Hungarian algorithm is the optimum SA solutions for maximizing the SNR of all users in the single-cell OFDMA system employing the channel-inverse power-allocation. Furthermore, as shown in [23, 30], the Hungarian algorithm is able to closely solve the problem of (24). Therefore, the benchmark for our SA can be obtained by independently operating the Hungarian algorithm at each BS, which corresponds to the benchmark-SA. However, the BenchmarkSA algorithm still has very high complexity, especially, when the number of users in the system is large.

\section{B. Benchmark of Distributed Spreading Code Allocation: Benchmark-CA}

After the SA, our distributed CA is carried out in each cell, aiming at maximizing the sum rate of every co-subcarrier user group, as shown in (16). However, the problem of (16) for the $\mathrm{CA}$ is a mixed integer nonlinear non-convex problem, which is hard to find the optimum solution. Moreover, directly solving (16) requires the BSs to exchange the full ICI information of users in different cells, which is not practical in real systems. Because of the above issues, we convert the problem in (16) to the concave problems of (25) and (27) by relaxing the constraint of (11) and reforming the users' rate expressions in (18), in order to study the benchmark performance of the system employing distributed CA. Specifically, the benchmark for our distributed CA, i.e. benchmark-CA, can be obtained by solving the relaxed problems of (25) and (27), which, for our three-cell system, is implemented in three stages by BSs $u, u^{\prime}$ and $u^{\prime \prime}$ $\left(u \neq u^{\prime} \neq u^{\prime \prime} \in \mathcal{U}\right)$ successively, when users' ICI information is not exchanged among the BSs. In detail, during the first stage, BS $u$ randomly allocates the codes to its users due to its lack of the knowledge about the CA results of the other cells. After the CA in the $u$ th cell, the users in cells $u^{\prime}$ and $u^{\prime \prime}$ are able to measure the ICI from cell $u$, which are informed to their BSs. Then, during the second stage, BS $u^{\prime}$ can carry out the CA based on the CA results of cell $u$, by solving the optimization problem 
given by

$$
\begin{aligned}
& \left\{\mathcal{C}_{m}^{\left(u^{\prime}\right)}\right\}^{*}= \\
& \arg \max _{\left\{\mathcal{C}_{m}^{\left(u^{\prime}\right)}\right\}}\left\{\sum_{k^{\prime} \in \mathcal{F}_{m}^{\left(u^{\prime}\right)}} R_{k^{\prime}}^{\left(u^{\prime}\right)} \mid\left\{\mathcal{C}_{m}^{(u)}, \mathcal{S}_{m}^{\left(u^{\prime}\right)}\right\}^{*}\right\}, \forall m \in \mathcal{M}
\end{aligned}
$$

subject to the constraints of (12), (13) with superscript $u$ substituted by $u^{\prime}$, and the relaxed constraint of

$$
0 \leq c_{k^{\prime}, n}^{\left(u^{\prime}\right)} \leq 1, \quad \forall k^{\prime} \in \mathcal{F}_{m}^{\left(u^{\prime}\right)}, \forall n \in \mathcal{N} .
$$

Following the CA in cell $u^{\prime}$, the users in cell $u^{\prime \prime}$ can now inform BS $u^{\prime \prime}$ the ICI from the other two BSs. Therefore, during the last stage, BS $u^{\prime \prime}$ can operate its CA with the knowledge of the ICI from both BSs $u$ and $u^{\prime}$, by solving the optimization problem of

$$
\begin{aligned}
& \left\{\mathcal{C}_{m}^{\left(u^{\prime \prime}\right)}\right\}^{*}= \\
& \arg \max _{\left\{\mathcal{C}_{m}^{\left(u^{\prime \prime}\right)}\right\}}\left\{\sum_{k^{\prime \prime} \in \mathcal{F}_{m}^{\left(u^{\prime \prime}\right)}} R_{k^{\prime \prime}}^{\left(u^{\prime \prime}\right)} \mid\left\{\mathcal{C}_{m}^{(u)}, \mathcal{C}_{m}^{\left(u^{\prime}\right)}, \mathcal{S}_{m}^{\left(u^{\prime \prime}\right)}\right\}^{*}\right\}, \forall m \in \mathcal{M}
\end{aligned}
$$

subject to the constraints in (12), (13) with superscript $u$ substituted by $u^{\prime \prime}$, and the relaxed constraint of

$$
0 \leq c_{k^{\prime \prime}, n}^{\left(u^{\prime \prime}\right)} \leq 1, \quad \forall k^{\prime \prime} \in \mathcal{F}_{m}^{\left(u^{\prime \prime}\right)}, \forall n \in \mathcal{N} .
$$

Furthermore, in order to make the problems in (25) and (27) the concave problems, the rates $R_{k^{\prime}}^{\left(u^{\prime}\right)}$ and $R_{k^{\prime \prime}}^{\left(u^{\prime \prime}\right)}$ are evaluated by the formulas of (29) and (30), which are equivalent to (18) by our simulation under the successive CA. In (30), $\tilde{I}_{k^{\prime}, m, n}^{(u)}$ is the ICI from BS $u$ to user $k^{\prime}$ in cell $u^{\prime}$ assigned subcarrier $m$ and code $n$ assigned. $\bar{I}_{k^{\prime}}^{\left(u^{\prime \prime}\right)}$ is the average ICI from BS $u^{\prime \prime}$, which is evaluated as $\bar{I}_{k^{\prime}}^{\left(u^{\prime \prime}\right)}=\left(\sum_{j \in \mathcal{F}_{m}^{\left(u^{\prime \prime}\right)}} I_{k^{\prime}, j, m}^{\left(u^{\prime \prime}\right)}\right) / N$, since the CA in cell $u^{\prime \prime}$ has not been done yet. Similarly, in (30), $\tilde{I}_{k^{\prime \prime}, n}^{(u)}$ and $\tilde{I}_{k^{\prime \prime}, n}^{\left(u^{\prime}\right)}$ count the ICI from BSs $u$ and $u^{\prime}$.

The CA problems in (25) and (27) are concave problems associated with the constraints in (12), (13), and the rates in (29), (30). The corresponding proofs are given in the Appendix. Therefore, there are many methods, such as the interior point method [32], that can be employed to solve the problems of (25) and (27). However, in order to solve the problems in (25) and (27), BSs $u$ and $u^{\prime}$ need to know the analog ICI information of their users, which may become challenging for the feedback channels in practice if there are a lot of users. In order to mitigate this demand, in this paper, we propose the RAIM scheme including the heuristic CA algorithm, which only requires the bit-valued ICI information by the BSs.

\section{Benchmark of Intercell Interference Mitigation: Benchmark-IM}

After the SA and CA, we can derive the centralized benchmark-IM algorithm, which can find the optimum solution to the problem of (19). In order to derive the optimum solution, the benchmark-IM carries out the exhaustive search with the knowledge of full ICI information for cell-edge users, and its principles can be summarized in Algorithm 1.
(Algorithm 1) Benchmark of ICI Mitigation: Benchmark-IM

1: Initialization: $\mathcal{B}_{m, n}=\left\{k \mid k \in \tilde{K}^{(u)} \cap \mathcal{F}_{m}^{(u)} \cap \mathcal{V}_{n}^{(u)}, \forall u\right\}$, $d_{m, n}^{(u)}=k$ if $\mathcal{F}_{m}^{(u)} \cap \mathcal{V}_{n}^{(u)}=\{k\}, \forall m \in \mathcal{M}, \forall u \in \mathcal{U}$;

2: $\quad$ For subcarrier $m=0, \ldots, M-1 \&$ code $n=0, \ldots, N-1$ 3: $\quad$ If $\mathcal{B}_{m, n} \neq \emptyset$

4: $\quad$ Compute sum rates of all optional decisions (OPs) with power off only:

$O P(1)$ Power off for one user:

$\hat{d}_{m, n}^{(u)}=-1, \hat{d}_{m, n}^{\left(u^{\prime}\right)}=d_{m, n}^{\left(u^{\prime}\right)}, \hat{d}_{m, n}^{\left(u^{\prime \prime}\right)}=d_{m, n}^{\left(u^{\prime \prime}\right)}, \forall u$,

$O P(2)$ Power off for two users:

$\hat{d}_{m, n}^{(u)}=\hat{d}_{m, n}^{\left(u^{\prime}\right)}=-1, \hat{d}_{m, n}^{\left(u^{\prime \prime}\right)}=d_{m, n}^{\left(u^{\prime \prime}\right)}, \forall u ;$

5: Compute sum rates of all the OPs with cooperation only: $O P(3)$ Cooperation between two BSs:

$\hat{d}_{m, n}^{(u)}=\hat{d}_{m, n}^{\left(u^{\prime}\right)}=d_{m, n}^{(u)}, \hat{d}_{m, n}^{\left(u^{\prime \prime}\right)}=d_{m, n}^{\left(u^{\prime \prime}\right)}, \forall u$,

$O P(4)$ Cooperation among three BSs:

$\hat{d}_{m, n}^{(i)}=d_{m, n}^{(u)}, \forall i \in\left\{u, u^{\prime}, u^{\prime \prime}\right\}, \forall u$;

6: $\quad$ Compute sum rates of all the OPs with both power off and cooperation:

$O P(5)$ Power off for one user, cooperation for one user:

$\hat{d}_{m, n}^{(u)}=\hat{d}_{m, n}^{\left(u^{\prime}\right)}=d_{m, n}^{(u)}, \hat{d}_{m, n}^{\left(u^{\prime \prime}\right)}=-1, \forall u ;$

7: $\quad$ Identify the best decision:

$\quad\left\{d_{m, n}^{(u)}, \forall u\right\}=\max _{\left\{\forall \hat{d}_{m, n}^{(u)}, \forall u\right\}}\left\{\sum_{k \in \mathcal{B}_{m, n}} R_{k}\right\}$;
8: $\quad$ End
9: $\quad$ End

In detail, under the benchmark-IM, we assume that a control unit (CU) collects the ICI information of all cell-edge users in the three cells. Then, the CU makes the best ICI mitigation decisions, which are informed to the BSs. The decisions are made independently for the cell-edge user groups of each containing three co-subcarrier-code users. For a cell-edge user group, the CU finds the best mitigation decision achieving the highest sum rate by exhaustively searching the 21 possible decisions, as stated in lines 3-8 of Algorithm 1. However, the benchmark-IM requires analog ICI information of all cell-edge users for decision making, which is required to be sent to the CU. In this case, the benchmark-IM may impose a heavy complexity burden on the backhaul network, especially when there is a big number of cell-edge users. Therefore, in Section IV, we propose the distributed ICI mitigation algorithm under the RAIM scheme, which does not require the BSs to know any ICI information of users.

\section{Novel Distributed Resource Allocation ASSISTED BY ICI MITIGATION}

In this section, we propose a novel low-complexity heuristic scheme, namely RAIM, which can approximate the benchmark scheme in Section III. The proposed RAIM is a fully distributed scheme for the resource allocation and ICI mitigation in the considered multicell system, and it does not require the BSs to share any channel information. The fully distributed RAIM scheme consists of the SA, CA and ICI mitigation algorithms, which are referred to as the RAIM-SA, the RAIM-CA and the RAIM-IM. Let us now first discuss the RAIM-SA algorithm.

\section{A. RAIM's Subcarrier Allocation: RAIM-SA}

The RAIM-SA algorithm motivates to find a promising suboptimum solution to the problem of (24), by maximizing the best SNR of users as well as the worst SNR of users. The 


$$
\begin{aligned}
& R_{k^{\prime}}^{\left(u^{\prime}\right)}=\sum_{n \in \mathcal{N}} c_{k^{\prime}, n}^{\left(u^{\prime}\right)} \log _{2}\left(1+\frac{\left|h_{k^{\prime}, m}^{\left(u^{\prime}\right)}\right|^{2}}{\sum_{n \in \mathcal{N}} c_{k^{\prime}, n}^{\left(u^{\prime}\right)} \tilde{I}_{k^{\prime}, m, n}^{(u)}+\bar{I}_{k^{\prime}}^{\left(u^{\prime \prime}\right)}+2 \sigma^{2}}\right), k^{\prime} \in \mathcal{F}_{m}^{\left(u^{\prime}\right)}, \\
& R_{k^{\prime \prime}}^{\left(u^{\prime \prime}\right)}=\sum_{n \in \mathcal{N}} c_{k^{\prime \prime}, n}^{\left(u^{\prime \prime}\right)} \log _{2}\left(1+\frac{\left|h_{k^{\prime \prime}, m}^{\left(u^{\prime \prime}\right)}\right|^{2}}{\sum_{n \in \mathcal{N}} c_{k^{\prime \prime}, n}^{\left(u^{\prime \prime}\right)}\left(\tilde{I}_{k^{\prime \prime}, m, n}^{(u)}+\tilde{I}_{k^{\prime \prime}, m, n}^{\left(u^{\prime}\right)}\right)+2 \sigma^{2}}\right), k^{\prime \prime} \in \mathcal{F}_{m}^{\left(u^{\prime \prime}\right)} .
\end{aligned}
$$

(Algorithm 2) Stage I of RAIM-SA: Candidate Searching

\section{(For BS $u, \forall u \in \mathcal{U}$ )}

1: $\quad$ Initialization: $\mathcal{F}_{m}^{(u)}=\mathcal{U}_{m}^{(u)}=\emptyset, \forall m \in \mathcal{M} ; \mathcal{Q}_{k}^{(u)}=\emptyset$, $\mathcal{M}_{k}^{(u)}=\mathcal{M}, \forall k \in \mathcal{K}^{(u)}$

\section{2: $\quad$ Repeat}

3: $\quad$ Each user identifies an unselected subcarrier having the best subchannel quality as a candidate:

(E2.1): $m_{k}^{\prime}=\arg \max _{m \in \mathcal{M}_{k}^{(u)}}\left\{A_{k, m}^{(u)}\right\}, \forall k \in \mathcal{K}^{(u)}$;

4: Update: $\begin{aligned} \text { (D2.1): } & \mathcal{U}_{m_{k}^{\prime}}^{(u)} \leftarrow \mathcal{U}_{m_{k}^{\prime}}^{(u)} \cup\{k\}, \mathcal{Q}_{k}^{(u)} \leftarrow \mathcal{Q}_{k}^{(u)} \cup\left\{m_{k}^{\prime}\right\}, \\ & \forall k \in \mathcal{K}^{(u)},\end{aligned}$

(D2.2): $\mathcal{M}_{k}^{(u)} \leftarrow \mathcal{M}_{k}^{(u)}-\left\{m_{k}^{\prime}\right\}, \forall k \in \mathcal{K}^{(u)}$;

5: $\quad$ Condition check:

(C2.1): $\left|\mathcal{U}_{m}^{(u)}\right| \geq N, \forall m \in \mathcal{M}$;

6: $\quad$ Until $\mathrm{C} 2.1=$ true

RAIM-SA has two stages, and is independently operated by each BS. During Stage I, the algorithm searches the candidates (including the subcarriers and users) having the best subchannel qualities. During Stage II, it allocates the candidates in the way that completes the allocation with the minimum number of candidates required. The principles of the RAIM-SA are summarized in Algorithms 2 and 3.

In Stage I given by Algorithm 2, it iteratively searches the best candidates in each cell. During an iteration, each user in cell $u$ identifies a candidate subcarrier which has not been selected and has the best subchannel quality. For the example in (E2.1), user $k$ finds that subcarrier $m_{k}^{\prime}$ is a candidate, and user $k$ is also seen as a candidate of subcarrier $m_{k}^{\prime}$. Then in line 4, BS $u$ updates the candidate sets $\mathcal{U}_{m}^{(u)}$ and $\mathcal{Q}_{k}^{(u)}$, which contain the candidate indexes, respectively, for subcarrier $m$ and user $k$ in cell $u$. At the end of each iteration, the algorithm checks if enough candidates are found, i.e. Condition (C2.1) is satisfied. The algorithm proceeds to Stage II when each subcarrier has at least $N$ candidate users, which is required by our allocation.

In Stage II, one candidate subcarrier is allocated to one candidate user in each iteration. The RAIM-SA algorithm motivates to complete the allocation in Stage II with the minimum number of candidates. As shown in Algorithm 3, during an iteration it first tries to finds the candidate which only has the fixed allocation option/options but no other options, as shown in lines 3-6. For instance, in line 3, it finds that subcarrier $m^{*}$ has to be assigned to its candidate user $k^{*}$. This is because, as shown in (E3.1), the number of the candidate users for subcarrier $m^{*}$ is equal to its possible number of allocations. Similarly, in line 5 , user $k^{*}$ has the only one candidate, i.e. subcarrier $m^{*}$. When the fixed allocation is unavailable, the allocation of the candidates having more than one allocation options follows the max-min method, which aims to maximize the minimum subchannel quality of the candidate assigned. In
(Algorithm 3) Stage II of RAIM-SA: Candidate Assigning

\section{(For BS $u, \forall u \in \mathcal{U}$ )}

1: $\quad$ Initialization: $\tilde{\mathcal{M}}=\mathcal{M}, \tilde{K}^{(u)}=\tilde{K}^{(u)}$;

2: $\quad$ Repeat

(Fixed Allocation)

3: Identify subcarrier $m^{*}$ with fixed allocation option/options and find its candidate user $k^{*}$ :

(E3.1): $m^{*} \in \check{\mathcal{M}}=\left\{m|| \mathcal{U}_{m}^{(u)}|=N-| \mathcal{F}_{m}^{(u)} \mid, m \in \tilde{\mathcal{M}}\right\}$, $k^{*} \in \mathcal{U}_{m^{*}}^{(u)}$;

4: $\quad$ If $\check{\mathcal{M}}=\emptyset$

5: $\quad$ Identify user $k^{*}$ having candidate subcarrier $m^{*}$ only: (E3.2): $k^{*} \in \check{\mathcal{K}}^{(u)}=\left\{k|| \mathcal{Q}_{k}^{(u)} \mid=1, k \in \tilde{\mathcal{K}}^{(u)}\right\}$, $m^{*} \in \mathcal{U}_{k^{*}}^{(u)}$

6: $\quad$ End

(Max-Min Allocation)

7: $\quad$ If $\check{\mathcal{M}}=\emptyset \& \check{\mathcal{K}}^{(u)}=\emptyset$

8: $\quad$ Identify user $k^{*}$ having the minimum worst candidate subchannel quality:

(E3.3): $k^{*}=\arg \min _{k \in \tilde{\mathcal{K}}(u)}\left\{\min _{m \in \mathcal{Q}_{k}^{(u)}}\left\{A_{k, m}^{(u)}\right\}\right\}$;

9: $\quad$ Find user $k^{*}$ the best candidate subcarrier $m^{*}$ having the highest subchannel quality:

\section{0: End} (E3.4): $m^{*}=\arg \max _{m \in \mathcal{Q}_{k^{*}}^{(u)}}\left\{A_{k^{*}, m}^{(u)}\right\}$;

11: Allocate subcarrier $m^{*}$ to candidate user $k^{*}$ : $\mathcal{F}_{m^{*}}^{(u)} \leftarrow \mathcal{F}_{m^{*}}^{(u)} \cup\left\{k^{*}\right\}$

12: Update:

(D3.1): $\mathcal{U}_{m^{*}}^{(u)} \leftarrow \mathcal{U}_{m^{*}}^{(u)}-\left\{k^{*}\right\} ; \mathcal{Q}_{k^{*}}^{(u)} \leftarrow \mathcal{Q}_{k^{*}}^{(u)}-\left\{m^{*}\right\}$,

(D3.2): $\tilde{\mathcal{K}}^{(u)} \leftarrow \tilde{\mathcal{K}}^{(u)}-\left\{k^{*}\right\}$

13: Condition check:

$$
\tilde{\mathcal{M}} \leftarrow \tilde{\mathcal{M}}-\left\{m^{*}\right\} \text { if }\left|\mathcal{F}_{m}^{(u)}\right|=N \text {; }
$$

(C3.1): $\left|\mathcal{U}_{m}^{(u)}\right| \geq N-\left|\mathcal{F}_{m}^{(u)}\right|, \forall m \in \tilde{\mathcal{M}}$

$$
\&\left|\mathcal{Q}_{k}^{(\bar{u})}\right| \geq 1, \forall k \in \tilde{\mathcal{K}}^{(u)} \text {; }
$$

14: Until $\tilde{\mathcal{M}}=\emptyset \quad$ or $\quad(\mathrm{C} 3.1)=$ false

15: $\quad$ Go back to line 2 of Stage I if $(\mathrm{C} 3.1)=$ false;

(E3.3), among the remaining users in $\mathcal{K}^{(u)}$, user $k^{*}$ is identified since it has the minimum worst candidate subchannel quality. Then it finds the best candidate subcarrier for user $k^{*}$, which is subcarrier $m^{*}$ having the highest subchannel quality. Once having identified the candidates, it carries out the corresponding allocation, which is given in line 11 . The algorithm completes the candidate allocation when allocation requirement is met. However, when there are not enough candidates for remaining allocation, i.e. Condition (C3.1) is unsatisfied, the algorithm goes back to Stage I to add more candidates.

\section{B. RAIM's Code allocation: RAIM-CA}

After carrying out the SA, the RAIM scheme operates the distributed CA, i.e. the RAIM-CA algorithm. The RAIM-CA motivates to approach the performance of the benchmark-CA algorithm. It can find the low-complexity sub-optimum solutions to the problems of (25) and (27) by minimizing the ICI 
of each co-subcarrier user group in each cell. The RAIM-CA is carried out in three stages successively and independently by the three BSs. Similar to the benchmark-CA, during the first stage, BS 0 randomly allocates codes to its users. During the second and third stages, the CAs of cells 1 and 2 follows the principles in Algorithm 4 based on the knowledge of the bitvalued ICI (bICI) information of intracell users.

Shown by Algorithm 4, the CA is carried out independently for each co-subcarrier user group containing $N$ users, and one code is allocated to a user during each of $N$ iterations. First of all, the users estimate their ICI from the cell/cells whose CA has/have been done in previous stage/stages. Then the users inform its BS the bICI for the allocation. For the example shown in (E4.1), $\hat{I}_{k^{\prime}, k, m}^{(u)}$ is the bICI received by user $k^{\prime}$ from BS $u$, when assuming both users $k$ and $k^{\prime}$ in cells $u$ and $u^{\prime}$ are assigned subcarrier $m$. The bICI $\hat{I}_{k^{\prime}, k, m}^{(u)}$ can be given by

$$
\hat{I}_{k^{\prime}, k, m}^{(u)}= \begin{cases}0 & \text { if } I_{k^{\prime}, k, m}^{(u)}<I_{t}, \\ 1 & \text { if } I_{k^{\prime}, k, m}^{(u)} \geq I_{t}\end{cases}
$$

where $I_{t}$ is the threshold that defines if the ICI is small $\left(\hat{I}_{k^{\prime}, k, m}^{(u)}=0\right)$ or strong $\left(\hat{I}_{k^{\prime}, k, m}^{(u)}=0\right)$. Known from (E4.1), during the second stage, $\mathrm{BS} u^{\prime}\left(u^{\prime}=1\right)$ only makes use of its users' bICI from BS 0 , which is evaluated as $\tilde{I}_{k^{\prime}, k, m}^{(u)}=$ $\hat{I}_{k^{\prime}, k, m}^{(u)}=\{0,1\}$. By contrast, during the third stage, BS $u^{\prime}$ $\left(u^{\prime}=2\right)$ employs the combined bICI from both BSs 0 and 1 , which is evaluated as $\tilde{I}_{k^{\prime}, k, m}^{(u)}=\{0,1,2\}$. Specifically, when $\tilde{I}_{k^{\prime}, k, m}^{(u)}=2$, it means that user $k^{\prime}$ suffers strong ICI from the other two BSs, which is the case that the RAIM-CA motivates to avoid.

As shown in lines 5-10, the RAIM-CA identifies a co-code user pair for allocating a code during each iteration. In line 12, when the pair $\left(k_{u}^{*}, k_{u^{\prime}}^{*}\right)$ is identified, user $k_{u^{\prime}}^{*}$ is allocated code $n^{*}$ which has been allocated to user $k_{u}^{*}$ in a previous stage. Note that, during the third stage, it actually finds a pair of co-code users for the user in cell 2 , since the CAs of cells 0 and 1 have been done in previous stages. The algorithm motivates to find the user pair of small ICI, which contains the co-code user generating the small ICI to the user. Therefore, the algorithm first tries to identify a user pair of small ICI for fixed allocation. As the example shown in line 5, when the pair $\left(k, k^{\prime}\right)$ in $\tilde{\Theta}_{m, 0}^{\left(u, u^{\prime}\right)}$ is identified, it means user $k^{\prime}$ has only one co-code user available, i.e. user $k$, generating small ICI to user $k^{\prime}$. By contrast, when the pair $\left(k, k^{\prime}\right)$ in $\tilde{\Theta}_{m, 1}^{\left(u, u^{\prime}\right)}$ is identified, user $k$ generates strong ICI to all the users of subcarrier $m$ in cell $u^{\prime}$ except user $k^{\prime}$. Note that, in (E4.2), $\tilde{\mathcal{F}}_{m}^{\left(u^{\prime}\right)}$ contains the indexes of the users with subcarrier $m$ allocated in cell $u^{\prime}$ but without codes assigned, and $\tilde{\mathcal{V}}_{m}^{(u)}$ contains the indexes of available co-code users in cell $u$ for the users in $\tilde{\mathcal{F}}_{m}^{\left(u^{\prime}\right)}$. When the fixed allocation of small ICI is unavailable, i.e. $\tilde{\Theta}_{m}^{\left(u, u^{\prime}\right)}=\emptyset$, the RAIM-CA selects the user pair of small ICI that can avoid the maximum number of the allocation of strong ICI in future allocation, which is described by line 6 .

Furthermore, when there is no co-code user pair of small ICI found, i.e. $\Theta_{m}^{\left(u, u^{\prime}\right)}=\emptyset$, the RAIM-CA tries to identify a desired user pair containing the user, which suffers strong ICI from only one BS. The identification process for this given in lines 8-9 is
(Algorithm 4) RAIM's Code Allocation: RAIM-CA

(For BS $u^{\prime}=1,2$ )

1: $\quad$ Initialization: $\mathcal{V}_{m}^{\left(u^{\prime}\right)}=\emptyset, \tilde{\mathcal{V}}_{m}^{(u)}=\mathcal{V}_{m}^{(u)}, \tilde{\mathcal{F}}_{m}^{\left(u^{\prime}\right)}=\mathcal{F}_{m}^{\left(u^{\prime}\right)}$, $u=0, \forall m \in \mathcal{M}$;

2: $\quad$ For Subcarrier $m=0, \ldots, M-1$

3: Users allocated subcarrier $m$ inform BS $u^{\prime}$ bICI information $\left\{\hat{I}_{k^{\prime}, k, m}^{(u)}, k^{\prime}, k\right\}$, BS $u^{\prime}$ forms information $\left\{\tilde{I}_{k^{\prime}, k, m}^{(u)}, k^{\prime}, k\right\}$ :

(E4.1): $\tilde{I}_{k^{\prime}, k, m}^{(u)}=\sum_{i=0}^{u} \hat{I}_{k^{\prime}, k_{i}, m}^{(i)}, k \in \mathcal{F}_{m}^{(u)}, k_{i} \in \mathcal{F}_{m}^{(i)}$,

$$
\forall k^{\prime} \in \mathcal{F}_{m}^{\left(u^{\prime}\right)}
$$

\section{4: $\quad$ Repeat}

5: $\quad$ Identify a co-code user pair of small ICI $\left(k_{u}^{*}, k_{u^{\prime}}^{*}\right) \in \tilde{\Theta}_{m}^{\left(u, u^{\prime}\right)}$ for fixed allocation, and $\tilde{\Theta}_{m}^{\left(u, u^{\prime}\right)}=\tilde{\Theta}_{m, 0}^{\left(u, u^{\prime}\right)} \cup \tilde{\Theta}_{m, 1}^{\left(u, u^{\prime}\right)}$ which are defined in (E4.2), (E4.3) and (E4.4);

6: $\quad$ Find the co-code user pair of small ICI $\left(k_{u}^{*}, k_{u^{\prime}}^{*}\right)$ that avoids the maximum number of strong ICI assignments if $\tilde{\Theta}_{m}^{\left(u, u^{\prime}\right)}=\emptyset \& \Theta_{m}^{\left(u, u^{\prime}\right)} \neq \emptyset$ :

(E4.5): $\left(k_{u}^{*}, k_{u^{\prime}}^{*}\right)=\arg \max _{\left(k, k^{\prime}\right) \in \Theta_{m}^{\left(u, u^{\prime}\right)}}\left\{\left|\check{\mathcal{K}}_{k}^{(u)}\right|+\left|\check{\mathcal{K}}_{k^{\prime}}^{\left(u^{\prime}\right)}\right|\right\}$, (E4.6): $\check{\mathcal{K}}_{k}^{(u)}=\left\{j^{\prime} \mid \tilde{I}_{j^{\prime}, k, m}^{(u)} \neq 0, \forall j^{\prime} \in \tilde{\mathcal{F}}_{m}^{\left(u^{\prime}\right)}\right\}$,

$$
\check{\mathcal{K}}_{k^{\prime}}^{\left(u^{\prime}\right)}=\left\{j \mid \tilde{I}_{k^{\prime}, j, m}^{(u)} \neq 0, \forall j \in \tilde{\mathcal{V}}_{m}^{(u)}\right\} ;
$$

7: $\quad$ If $\Theta_{m}^{\left(u, u^{\prime}\right)}=\emptyset$

8: $\quad$ Identify a desired co-code user pair of strong ICI

$\left(k_{u}^{*}, k_{u^{\prime}}^{*}\right) \in \tilde{\Lambda}_{m}^{\left(u, u^{\prime}\right)}$ for fixed allocation, and

$\tilde{\Lambda}_{m}^{\left(u, u^{\prime}\right)}=\tilde{\Lambda}_{m, 0}^{\left(u, u^{\prime}\right)} \cup \tilde{\Lambda}_{m, 1}^{\left(u, u^{\prime}\right)}$, which are defined in (E4.7), (E4.8) and (E4.9);

9: $\quad$ Identify a desired co-code user pair of strong ICI $\left(k_{u}^{*}, k_{u^{\prime}}^{*}\right) \in \Lambda_{m}^{\left(u, u^{\prime}\right)}$ if $\tilde{\Lambda}_{m}^{\left(u, u^{\prime}\right)}=\emptyset$;

10: $\quad$ Select a co-code user pair of strong ICI $\left(k_{u}^{*}, k_{u^{\prime}}^{*}\right)$, where $k \in \tilde{\mathcal{V}}_{m}^{(u)}, k^{\prime} \in \tilde{\mathcal{F}}_{m}^{\left(u^{\prime}\right)}$ if $\Lambda_{m}^{\left(u, u^{\prime}\right)}=\emptyset ;$

\section{1: End}

12: Allocate code $n^{*}$ to user $k_{u^{\prime}}^{*}: \mathcal{V}_{m}^{\left(u^{\prime}\right)} \leftarrow \mathcal{V}_{m}^{\left(u^{\prime}\right)}\left(n^{*}\right) \cup\left\{k_{u^{\prime}}^{*}\right\}$, where $\mathcal{V}_{m}^{(u)}\left(n^{*}\right)=\left\{k_{u}^{*}\right\}$;

13: $\quad$ Update: $\tilde{\mathcal{V}}_{m}^{(u)} \leftarrow \tilde{\mathcal{V}}_{m}^{(u)}-\left\{k_{u}^{*}\right\}, \tilde{\mathcal{F}}_{m}^{\left(u^{\prime}\right)} \leftarrow \tilde{\mathcal{F}}_{m}^{\left(u^{\prime}\right)}-\left\{k_{u^{\prime}}^{*}\right\}$;

14: Until $\tilde{\mathcal{F}}_{m}^{\left(u^{\prime}\right)}=\emptyset$

15: End

16: $\quad$ Update: $u \leftarrow u+1$;

similar to that for a user pair of small ICI shown in lines 5-6. Finally, when a desired co-code user pair cannot be identified, as in line 10, the algorithm is forced to select a co-code user pair including the user, which suffers strong ICI from two BSs.

\section{RAIM's ICI Mitigation: RAIM-IM}

After the SA and CA, the RAIM scheme carries out the distributed ICI mitigation, namely RAIM-IM. The RAIM-IM algorithm motivates to find a promising sub-optimum solution to the problem of (19) in order to approach the performance of the benchmark-IM. The BSs successively and independently carry out the RAIM-IM for their cell-edge users, but they do not share any channel information. For the sake of comparison, we also extend the existing on-off power (OOP) ICI mitigation algorithm [28,33] to our multicell systems. Let us first briefly discuss the principles of the OOP algorithm under our systems.

The OOP algorithm $[28,33]$ employed by the OFDMA systems can efficiently mitigate ICI, which however does not employ BS cooperation. In a little more detail, the core principle of the OOP is to allow BSs to switch off the transmissions suffering strong ICI. In our three-cell MC DS-CDMA systems, the OOP is scheduled to be carried out by the BSs in three 
$(\mathrm{E} 4.2): \Theta_{m}^{\left(u, u^{\prime}\right)}=\left\{\left(k, k^{\prime}\right) \mid \tilde{I}_{k^{\prime}, k, m}^{(u)}=0, k \in \tilde{\mathcal{V}}_{m}^{(u)}, k^{\prime} \in \tilde{\mathcal{F}}_{m}^{\left(u^{\prime}\right)}\right\},(\mathrm{E} 4.3): \tilde{\Theta}_{m, 0}^{\left(u, u^{\prime}\right)}=\left\{\left(k, k^{\prime}\right) \mid \prod_{j \in \tilde{\mathcal{V}}_{m}^{(u)}, j \neq k} \tilde{I}_{k^{\prime}, j, m}^{(u)} \neq 0,\left(k, k^{\prime}\right) \in \Theta_{m}^{\left(u, u^{\prime}\right)}\right\}$,

(E4.4): $\tilde{\Theta}_{m, 1}^{\left(u, u^{\prime}\right)}=\left\{\left(k, k^{\prime}\right) \mid \prod_{j^{\prime} \in \tilde{\mathcal{F}}_{m}^{\left(u^{\prime}\right), j^{\prime} \neq k^{\prime}}} \tilde{I}_{j^{\prime}, k, m}^{(u)} \neq 0,\left(k, k^{\prime}\right) \in \Theta_{m}^{\left(u, u^{\prime}\right)}\right\}$.

$(\mathrm{E} 4.7): \Lambda_{m}^{\left(u, u^{\prime}\right)}=\left\{\left(k, k^{\prime}\right) \mid \tilde{I}_{k^{\prime}, k, m}^{(u)}=1, k \in \tilde{\mathcal{V}}_{m}^{(u)}, k^{\prime} \in \tilde{\mathcal{F}}_{m}^{\left(u^{\prime}\right)}\right\},(\mathrm{E} 4.8): \tilde{\Lambda}_{m, 0}^{\left(u, u^{\prime}\right)}=\left\{\left(k, k^{\prime}\right) \mid \tilde{I}_{k^{\prime}, j, m}^{(u)}=2, j \neq k, \forall j \in \tilde{\mathcal{V}}_{m}^{(u)},\left(k, k^{\prime}\right) \in \Lambda_{m}^{\left(u, u^{\prime}\right)}\right\}$

$(\mathrm{E} 4.9): \tilde{\Lambda}_{m, 1}^{\left(u, u^{\prime}\right)}=\left\{\left(k, k^{\prime}\right) \mid \tilde{I}_{j^{\prime}, k, m}^{(u)}=2, j^{\prime} \neq k^{\prime}, \forall j^{\prime} \in \tilde{\mathcal{F}}_{m}^{\left(u^{\prime}\right)},\left(k, k^{\prime}\right) \in \Lambda_{m}^{\left(u, u^{\prime}\right)}\right\}$.

(Algorithm 5) RAIM's ICI mitigation: RAIM-IM

(For Subcarrier $m, \forall m \in \mathcal{M}$ and Code $n, \forall n \in \mathcal{N}$ )

1: Initialization: $d_{m, n}^{(u)}=k, d_{m, n}^{\left(u^{\prime}\right)}=k^{\prime}, d_{m, n}^{\left(u^{\prime \prime}\right)}=k^{\prime \prime}$, where $\mathcal{V}_{m}^{(u)}(n)=k, \mathcal{V}_{m}^{\left(u^{\prime}\right)}(n)=k^{\prime}, \mathcal{V}_{m}^{\left(u^{\prime \prime}\right)}(n)=k^{\prime \prime} ; u \neq u^{\prime} \neq u^{\prime \prime}$, $\forall u^{\prime}, u^{\prime \prime} \in \mathcal{U}$;

2: $\quad$ For Stage $u=0,1,2$

3: $\quad$ User $k$ estimates its SIR $\eta_{k, m}^{(u)}$,

if $\eta_{k, m}^{(u)}<\eta_{t} \& d_{m, n}^{(u)}=k$, execute:

4: $\quad$ User $k$ informs BS $u$ the requirement of the cooperation from BS $u^{\prime}$ (or $\left.u^{\prime \prime}\right)$ if $(\mathrm{C} 5.1)=$ true (or $(\mathrm{C} 5.2)=$ true), (C5.1): $\tilde{\eta}_{k, m}^{(u)} \geq \eta_{t} \& I_{k, k^{\prime \prime}, m}^{\left(u^{\prime \prime}\right)}<I_{c} \leq I_{k, k^{\prime}, m}^{\left(u^{\prime}\right)}$, (C5.2): $\tilde{\eta}_{k, m}^{(u)} \geq \eta_{t} \& I_{k, k^{\prime}, m}^{\left(u^{\prime}\right)}<I_{c} \leq I_{k, k^{\prime \prime}, m}^{\left(u^{\prime \prime}\right)}$;

5: $\quad$ BS $u$ requests BS $u^{\prime}$ (or $u^{\prime \prime}$ ) for the cooperation for user $k$;

6: $\quad \mathrm{BS} u^{\prime}$ (or $u^{\prime \prime}$ ) accepts the cooperation for user $k: d_{m, n}^{\left(u^{\prime}\right)}=k$ (or $d_{m, n}^{\left(u^{\prime \prime}\right)}=k$ ), if BS $u^{\prime}$ (or $u^{\prime \prime}$ ) is available $d_{m, n}^{\left(u^{\prime}\right)}=k^{\prime}$ (or $\left.d_{m, n}^{\left(u^{\prime \prime}\right)}=k^{\prime \prime}\right)$;

7: $\quad$ If $\quad\left((\mathrm{C} 5.1)=\right.$ false and/or $\left.d_{m, n}^{\left(u^{\prime}\right)}=k^{\prime \prime}\right) \quad \&$ $\left((\mathrm{C} 5.2)=\right.$ false and/or $\left.d_{m, n}^{\left(u^{\prime \prime}\right)}=k^{\prime}\right)$

8: $\quad$ BS $u$ broadcasts message that it can provide cooperation; 9: $\quad$ BSs $u^{\prime}$ (or $u^{\prime \prime}$ ) confirms that user $k^{\prime}$ (or $k^{\prime \prime}$ ) requires the cooperation from BS $u$ if $($ C5.3) $=$ true (or $($ C5.4) $=$ true), (C5.3): $d_{m, n}^{\left(u^{\prime}\right)}=k^{\prime} \& \tilde{\eta}_{k^{\prime}, m}^{\left(u^{\prime}\right)} \geq \eta_{t} \& I_{k^{\prime \prime}, k, m}^{(u)}<I_{c} \leq I_{k^{\prime}, k, m}^{(u)}$, (C5.4): $d_{m, n}^{\left(u^{\prime \prime}\right)}=k^{\prime \prime} \& \tilde{\eta}_{k^{\prime \prime}, m}^{\left(u^{\prime \prime}\right)} \geq \eta_{t} \& I_{k^{\prime}, k, m}^{(u)}<I_{c} \leq I_{k^{\prime \prime}, k, m}^{(u)}$;

10: $\quad$ BS $u$ accepts the cooperation for user $k^{\prime}: d_{m, n}^{(u)}=k^{\prime}$, if $\eta_{k^{\prime}, m}^{\left(u^{\prime}\right)}<\eta_{t}$. Otherwise, BS $u$ accepts the cooperation for user $k^{\prime \prime}: d_{m, n}^{(u)}=k^{\prime \prime}$;

11: $\quad$ BS $u$ switches off the transmission to user $k: d_{m, n}^{(u)}=-1$, if $($ C5.3 $)=$ false \& $($ C 5.4 $)=$ false;

12:

End

13: End

stages. The OOP algorithm makes the ICI mitigation decisions independently for the cell-edge user groups. During stage $u$ ( $u \in \mathcal{U}=\{0,1,2\}$ ), BS $u$ turns off the transmission to the user with poor SIR, such as user $k\left(\eta_{k}^{(u)}<\eta_{t}\right)$, where $\eta_{t}$ is the SIR threshold. By turning off the transmissions having poor SIR, it saves power for future transmissions, when the transmissions' communication qualities become improved. In addition, the ICI imposed by these transmissions with poor channel conditions on the other cells can also be removed.

By contrast, our RAIM-IM algorithm employs STBC based BS cooperation in addition to the strategy of power off, in order to achieve improved performance. However, the cooperation cost is mainly the increase of the complexity for exchanging users' data among BSs, and that the BSs have to stop transmitting information to some of their own users. Therefore, the RAIM-IM aims to maximize the pay-off from BS cooperation, while minimizing the cost. The principles of the RAIM-IM are summarized in Algorithm 5.

The RAIM-IM makes the ICI mitigation decisions for the cell-edge user groups of each containing three co-subcarriercode users within three stages. As the example of Algorithm 5 , subcarrier $m$ and code $n$ are assumed in line 1 to allocate to users $k, k^{\prime}$ and $k^{\prime \prime}$ in cells $u, u^{\prime}$ and $u^{\prime \prime}$. During stage $u$, user $k$ informs BS $u$ the requirement of ICI mitigation if it finds that its SIR is below the threshold, i.e. $\eta_{k, m}^{(u)}<\eta_{t}$. Then the mitigation decision can be made by lines 3-14. The RAIMIM algorithm motivates to maximize the benefit from using BS cooperation. Hence, in line 4 user $k$ first checks if the conditions for cooperation are met, i.e. (C5.1) and/or (C5.2) are satisfied. User $k$ requests the cooperation from BS $u^{\prime}$ when (C5.1) is true. There are two conditions included in (C5.1). First, the new SIR of user $k$ after the cooperation, i.e. $\tilde{\eta}_{k, m, n}^{(u)}$, should exceed the SIR threshold, which is $\eta_{k, m, n}^{(u)} \leq \eta_{t} \leq \tilde{\eta}_{k, m, n}^{(u)}$. Second, only one neighboring imposes strong ICI on user $k$, i.e. $I_{k, k^{\prime \prime}, m}^{\left(u^{\prime \prime}\right)}<I_{c} \leq I_{k, k^{\prime}, m}^{\left(u^{\prime}\right)}$ or $I_{k, k^{\prime}, m}^{\left(u^{\prime}\right)}<I_{c} \leq I_{k, k^{\prime \prime}, m}^{\left(u^{\prime \prime}\right)}$. The philosophy behind the second condition can be explained as follows. If BSs $u^{\prime}$ and $u$ set up the cooperative transmission for user $k$, the SIRs of the three users become

$$
\begin{gathered}
\tilde{\eta}_{k, m}^{(u)}=\frac{\left|h_{k, m}^{(u)}\right|^{2}+I_{k, k^{\prime}, m}^{\left(u^{\prime}\right)}}{I_{k, k^{\prime \prime}, m}^{\left(u^{\prime \prime}\right)}}, \tilde{\eta}_{k^{\prime}, m}^{\left(u^{\prime}\right)}=0 \\
\tilde{\eta}_{k^{\prime \prime}, m}^{\left(u^{\prime \prime}\right)}=\frac{\left|h_{k^{\prime \prime}, m}^{\left(u^{\prime \prime}\right)}\right|^{2}}{I_{k^{\prime \prime}, k, m}^{(u)}+I_{k^{\prime \prime}, k^{\prime}, m}^{\left(u^{\prime}\right)}} .
\end{gathered}
$$

Known from (32), the SIR of user $k$ can be significantly improved, when $I_{k, k^{\prime}, m}^{\left(u^{\prime}\right)}$ is large but $I_{k, k^{\prime \prime}, m}^{\left(u^{\prime \prime}\right)}$ is small. In this case, the sum rate of the three users is most probably increased owing to making use of the strong ICI of $I_{k, k^{\prime}, m}^{\left(u^{\prime}\right)}$. By contrast, when both $I_{k, k^{\prime}, m}^{\left(u^{\prime}\right)}$ and $I_{k, k^{\prime \prime}, m}^{\left(u^{\prime \prime}\right)}$ are very weak or very strong, the sum rate of the three users contributed by this BS cooperation is insignificant. As stated in line 6, BS $u^{\prime}$ accepts the cooperation request for user $k$ when it is available $d_{m, n}^{\left(u^{\prime}\right)}=k^{\prime}$, in other words, when it has not established a cooperation with another BS. Note that, when two BSs agree on a cooperation, the BS sends its user's data information to the cooperating BS. For the example, BS $u$ sends the data symbol of user $k$ to BS $u^{\prime}$ so that the two BSs carry out the STBC transmission to user $k$.

When the conditions in line 7 are met, it means that neither BS $u^{\prime}$ nor BS $u^{\prime \prime}$ can provide the cooperation for user $k$. Then the RAIM-IM algorithm requests BS $u$ to provide cooperation for users $k^{\prime}$ or $k^{\prime \prime}$ in the other cells, which follows the process in lines 8-10. In line 9 BS $u$ broadcasts the message of the availability of cooperation from itself. Then BSs $u^{\prime}$ and $u^{\prime \prime}$ ask their users to check if the conditions for the cooperation, i.e. (C5.3) or (C5.4), can be satisfied. Note that, the conditions of (C5.3) and (C5.4) are similar to those of (C5.1) and (C5.2), and the philosophies have been explained above. It is worth noting 
that, when both the BSs request the cooperation from $\mathrm{BS} u$, the algorithm always prefers to set up the cooperation for the user with poor SIR. As shown in line 10, BS $u$ accepts the cooperation for user $k^{\prime}$, since $\eta_{k^{\prime}, m}^{\left(u^{\prime}\right)}<\eta_{t}$. However, when no cooperation can be set up, BS $u$ has to turn off the transmission to user $k$, shown by line 11 .

\section{CharaCteristic AND COMPleXity ANAlysis}

In this section, we analyze the characteristics and the complexity of the RAIM scheme as well as the benchmark scheme.

\section{A. Characteristic Analysis}

The proposed benchmark scheme is obtained by solving and closely solving the formulated problems in (14), (16) and (19), which are respectively for the distributed SA and CA, as well as the ICI mitigation. For the considered multicell DS-CDMA system, the benchmark scheme proposes a novel strategy of resource allocation and ICI mitigation. It proposes that distributed SA is first carried out for maximizing intracell subchannel quality, then distributed $\mathrm{CA}$ is operated for maximizing the sum rate of the co-subcarrier users, and ICI mitigation is finally implemented for maximizing the sum rate of cell-edge users. The proposed strategy can be efficiently employed by practical multicell communication systems owing to the various advantages summarized as follows. First, the strategy employs distributed resource allocation which can quickly respond to various wireless environments, and it can also be easily extended to large systems with the minimum amount of cost needed. Second, the proposed strategy motivates to keep backhaul burden as low as possible. Third, the proposed strategy also aims to minimize the load on intracell feedback channels, where very limited ICI information is only required to transmit.

The RAIM scheme follows the novel strategy proposed by the benchmark scheme, and it also motivates to achieve the optimization objectives of the benchmark. By contrast, the RAIM scheme is designed to approach the performance of the benchmark scheme, while lowering implementation complexity and further minimizing backhaul burden as well as decreasing the cost of feedback channels. Let us now discuss the characteristics of the RAIM's SA, CA and ICI mitigation algorithms, and compare them with those of the benchmark scheme.

The RAIM-SA is a fully distributed SA algorithm, which requires much lower complexity than the benchmark-SA and the other existing SA algorithms as analyzed in Section V-B. The complexity required by the RAIM-SA is directly proportional to the number of times $S$ for operating Stage II to complete allocation. Hence, in Table I, we summarize the average values of $\hat{S}$ according to the simulation considering the various cases. Seen from Table I, $S \leq 2$ is always very small even when the values of $M$ and $N$ are big. In contrast to the benchmarkSA and the other existing SA algorithms, the RAIM-SA only requires each BS to know the intracell CSI of a part of its users, which guarantees very small signaling burden on feedback channels and very low implementation complexity. From Table I, we also observe the RAIM-SA algorithm requires a BS to know the intracell CSI of less than $50 \%$ users, i.e. $\rho<0.5$, for most scenarios. Due to the above advantages, the RAIMSA can be efficiently used for the SA in other multicarrier systems which employ very high number of subcarriers, such as OFDMA systems.

As suggested by the benchmark-CA, the RAIM-CA independently and successively operate the CA for the cells in two stages, which guarantees very low implementation complexity. By contrast, the RAIM-CA motivates to maximize the sum rate of co-subcarrier users by avoiding strong ICI as many as possible in order to efficiently mitigate ICI. Furthermore, for minimizing the signal burden on intracell feedback channels, the RAIM-CA only requires a BS to carry out the CA based on the binary information about a part of ICI for its users.

Both the benchmark-IM and RAIM-IM algorithms aim to maximize the sum rate of the cell-edge users with the aid of BS cooperation and power off. The two algorithms establish the cooperation between two BSs instead of that among three BSs in the considered three-cell systems, which keeps the cooperation cost and implementation complexity as low as possible. The benchmark-IM requires the BSs to exchange the ICI information of cell-edge users, which can be avoided by the RAIM-IM in order to further lower the backhaul resources. Moreover, the benchmark-IM is able to find the optimal ICI mitigation solutions by means of the exhaustive search with a relatively high complexity required. By contrast, the distributed RAIM-IM algorithm mainly motivates to minimize the implementation complexity, while finding the suboptimal ICI mitigation solutions by maximizing the benefit of BS cooperation.

The proposed benchmark and RAIM schemes can be modified for deployment in practical systems which may have a large number of cells and users. First, the proposed SA algorithms will be directly used in practical systems, since they are fully distributed algorithms based on the intracell CSI only. Note that in practical systems, the RAIM-SA algorithm can find better suboptimal SA solutions, since more number of subcarriers employed by practical systems allows the SA to exploit higher selecting diversity. Second, similar to the three-cell case, the CA is also carried out cell by cell in practical scenarios. The CA for a cell needs to consider the ICI effect from the cells that have done $\mathrm{CA}$ in previous stages. Owing to the structure of the practical cellular systems, one user can usually simultaneously receive strong ICI from two neighboring cells at most, which happens when a user is located near the borders of three cells. Hence, the cells operating the CA after the second stage can consider the strongest two ICI effects only on each user, which will release a lot signal burden on feedback channels. Third, the proposed ICI mitigation algorithms can be directly applied to practical systems, where each cell-edge user group contains only three co-subcarrier-code users generating strong ICI to each other. However, there is still a possibility that one user is simultaneously a member of two or more cell-edge user groups. In this case, the ICI mitigation algorithms can be modified to simply switch off the transmission to a user belonging to two or more cell-edge user groups.

\section{B. Complexity Analysis}

In this section, we analyze the complexity of the benchmark and the RAIM schemes. The complexity mainly reflects the 
TABLE I: Values $(S, \rho)$ are average number of times for operating Stage II, and average percentage of users with CSI required.

\begin{tabular}{|c||c|c|c|c|}
\hline$N$ & 4 & 8 & 16 & 32 \\
\hline \hline 1 & $(1.012,0.302)$ & $(1.134,0.313)$ & $(1.257,0.337)$ & $(1.389,0.408)$ \\
\hline 2 & $(1.018,0.428)$ & $(1.140,0.421)$ & $(1.361,0.468)$ & $(1.546,0.519)$ \\
\hline 4 & $(1.026,0.546)$ & $(1.120,0.528)$ & $(1.533,0.567)$ & $(1.788,0.648)$ \\
\hline 8 & $(1.049,0.669)$ & $(1.373,0.656)$ & $(1.751,0.672)$ & $(1.925,0.712)$ \\
\hline
\end{tabular}

number of comparisons required by the schemes. For our complexity analysis, we assume $M$ subcarriers and $N$ codes are used to support $U K$ users in the system, where $U=|\mathcal{U}|$ is the number of cells and $K=M N$.

The benchmark scheme employs the Hungarian algorithm for distributed SA, which requires $U\left(11 K^{3}+12 K^{2}+31 K\right) / 6$ comparisons for the multicell system [34]. The benchmark-CA algorithm is obtained by solving the concave problems of (25) and (27) with the aid of the interior point method. According to the complexity analyses for the interior point method in [3537], we readily know the benchmark-CA algorithm requires the computational complexity of at least $\mathcal{O}\left(M N^{4} L\right)$, where $L$ is the number of bits needed to represent the coefficients in the objective functions and the constraints. The RAIM-IM algorithm searches the best ICI mitigation decisions from all possible options for each cell-edge user group of each having $U$ co-subcarrier-code users. It needs $(U+1)^{U}\left(\left(\begin{array}{c}2 \\ U+1\end{array}\right)+1\right)$ comparisons for one cell-edge user group, since each user's IMD variable has $(U+1)$ states with the constraints of $(21)$ and (22). Hence, the complexity of the RAIM-IM is $\mathcal{O}\left(\left|\tilde{K}^{(u)}\right| U^{U}\right)$. In summary, the benchmark scheme has the complexity of $\mathcal{O}\left(\max \left\{U K^{3}, M N^{4} L,\left|\tilde{K}^{(u)}\right| U^{U}\right\}\right)$.

Let us now discuss the complexity of the RAIM scheme consisting of the RAIM-SA, RAIM-CA and the RAIM-IM algorithms. During Stage I of the RAIM-SA, it needs to find the candidates, which is equivalent to ordering $M$ number of subchannel qualities for each user. Hence, the number of comparisons required by this process is $2 K M \ln M$. In addition, the RAIM-SA demands $S M$ number of comparisons for the condition check in Stage I, where $S$ is given in Table I. During Stage II, the worst scenario requiring the highest complexity happens if that, each subcarrier is allocated to $(N-1)$ users during the first $(K-M)$ iteration via the max-min allocation. The first $(K-M)$ iterations requires $M(K-M)+(M+2)(M-$ $1) / 2+(K+2)(K-1) / 2$ comparisons for the searching process of the fixed allocation, and needs at most $K(S+1) \ln (S+1)$ comparisons for the max-min allocation. When accumulating the comparisons required by the two stages, the RAIM-SA in total requires the number of comparisons given by

$$
\begin{aligned}
\Phi_{\text {RAIM-SA }} \leq & 2 U K M \ln M+U S M(K-M+1) \\
& +\frac{U S(M+2)(M-1)}{2}+\frac{U S(K+2)(K-1)}{2} \\
& +U S K(S+1) \ln (S+1) .
\end{aligned}
$$

The RAIM-CA operates independently for the co-subcarrier user groups of each having $N$ users. Hence, let us analyze the complexity required by the $\mathrm{CA}$ for one user group. The worst scenario demanding the highest complexity happens if that, it can carry out the CA for a user pair of small ICI during each iteration. Known from Algorithm 4, the worst scenario
TABLE II: Complexity Comparison of Various Schemes.

\begin{tabular}{|c|c|}
\hline Algorithm & Complexity \\
\hline \hline Benchmark & $\mathcal{O}\left(\max \left\{U K^{3}, M N^{4} L,\left|\tilde{K}^{(u)}\right| U^{U}\right\}\right)$ \\
\hline RAIM & $\mathcal{O}\left(U S K^{2}\right)$ \\
\hline DRA-1 & $\mathcal{O}\left(U K^{2}\right)$ \\
\hline DRA-2 & $\mathcal{O}\left(U K^{2}\right)$ \\
\hline
\end{tabular}

always needs to identify all the user pairs of small ICI, which requires $2 N+(N+2)(N-1)$ comparisons. Note also that the fixed allocation of a small ICI pair is not available in the worst scenario. Hence, the algorithm needs to find the pair avoids the maximum number of strong ICI, which demands $(2 N+3)(N-$ 1) comparisons. Therefore, for the multicell system the RAIM$\mathrm{CA}$ in total requires the number of comparisons given by

$$
\begin{aligned}
\Phi_{\text {RAIM-CA }} \leq & (U-1)(2 K+2 M) \\
& +(U-1)(3 N+5)(K-M) .
\end{aligned}
$$

The RAIM-IM algorithm, presented in Algorithm 5, independently makes the ICI mitigation decisions for the cell-edge user groups. Hence, let us analyze the complexity required by one cell-edge user group. The worst scenario requiring the highest complexity happens if that, all the users' SIR is below the threshold, but no cooperation can be set up. During each stage, the algorithm first tries to find a neighboring BS's cooperation, then tries to provide the neighboring BS with the cooperation. Hence, to complete the above process, the complexity mainly comes from checking conditions (C5.1)-(C5.4), and checking the states of the IMD variables, and sending the requests. In total, the number of comparisons required by the RAIM-IM for the multicell system should satisfy

$$
\Phi_{\text {RAIM-IM }} \leq 4 U\left|\tilde{K}^{(u)}\right|+K .
$$

By combining the results in (33)-(35), we can readily know the RAIM scheme requires the complexity of $\mathcal{O}\left(U S K^{2}\right)$.

In Table II, we summarize the complexity required by different schemes. Note that, for the sake of comparison, in this paper we consider two existing distributed resource allocation (DRA) schemes, which are 1) DRA-1, and 2) DRA-2. The DRA-1 and DRA-2 schemes first operate the distributed SA based on the greedy algorithm [1] which has been widely used in various multicell multicarrier systems. Then, both the schemes carry out the random CA (RCA), since there is no CA proposed in existing literatures. After the SA and CA, the DRA-2 scheme uses the OOP algorithm [28] for ICI mitigation, while the DRA1 does not employ ICI mitigation for achieving low complexity and acquiring no backhaul resources. From Table II, we readily know that the RAIM scheme requires a much smaller complexity than the benchmark scheme. The RAIM's complexity is also similar to that of the low-complexity of DRA-1 and DRA-2, since the value of $S$ given by Table I is always very 
TABLE III: Simulation Parameters.

\begin{tabular}{|l|c|}
\hline Parameter & Value \\
\hline$U:$ Number of cells $[14,24,25]$ & 3 \\
\hline$K:$ Number of users in a cell & $64,16,8$ \\
\hline$M:$ Number of subcarriers & 16,8 \\
\hline$N:$ Number of codes & $8,4,1$ \\
\hline$\mu:$ Pathloss exponent $[5,8,38]$ & 4.0 \\
\hline$\Upsilon:$ Std. deviation of shadowing $[5,8,10,38]$ & $8(\mathrm{~dB})$ \\
\hline$\eta_{t}:$ SIR threshold & $-10,-9, \ldots, 2(\mathrm{~dB})$ \\
\hline$I_{t}:$ ICI threshold & $-20,-19, \ldots, 0(\mathrm{~dB})$ \\
\hline$I_{c}:$ ICI cooperation threshold & $-8,-7, \ldots, 8(\mathrm{~dB})$ \\
\hline
\end{tabular}

small. Furthermore, Fig. 2 compares the complexity of the various schemes in the three-cell systems, when considering the different scenarios. Observed from Fig. 2, the complexity required by the RAIM is always significant smaller than that of the benchmark. We further note that, as $N$ gets bigger, the complexity gap between the RAIM and benchmark increases while the gaps between the RAIM and DRA-1 as well as DRA2 decrease. From the above observations we can conclude that our RAIM scheme requires very low complexity, which makes it highly meaningful for practical implementation.

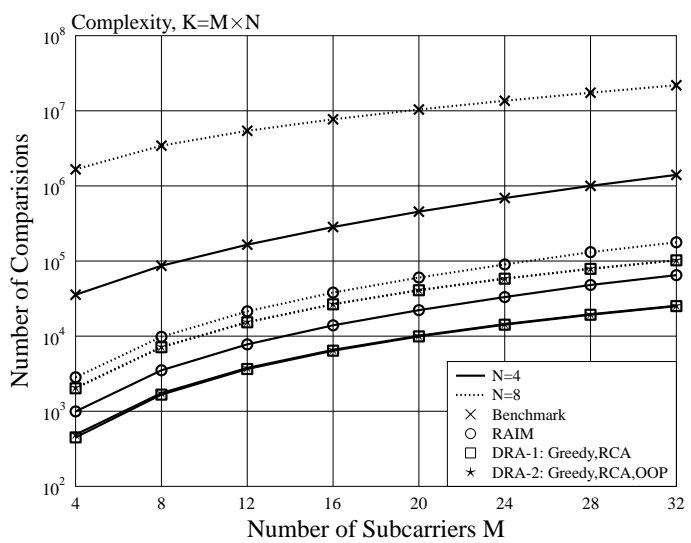

Fig. 2: Number of comparisons required by various algorithms.

\section{Vi. Performance Results}

In this section, we provide a range of simulation results for demonstrating the achievable SE performance of the multicell downlink MC DS-CDMA systems employing the proposed RAIM and benchmark schemes as well as other existing distributed resource allocation schemes. In our simulations, we assume that all subcarriers experience independent flat Rayleigh fast fading. Furthermore, the considered system consists of three cells, and each cell has $K=M N$ users supported by $M$ subcarriers and length- $N$ orthogonal spreading codes. The other main simulation parameters are summarized in Table III.

In Fig. 3, we show the SE per user performance of the proposed RAIM scheme versus the other resource allocation schemes employed by the systems. Here, the SE per user is obtained by the bandwidth normalized system's sum rate divided by the total number of users in the system. Fig. 3(a) assumes the number of subcarriers is equal to the number of codes, and we have the following observations. First, our RAIM scheme achieves the SE performance which is very close to that of the analytical benchmark scheme, and the tight performance gap stays unchanged regardless of the average SNR per symbol. Second, the RAIM scheme can significantly outperform the DRA-1 and DRA-2 schemes, and it becomes more advantageous over the existing schemes as the average SNR gets bigger. From the above observations, we can conclude that the RAIM scheme provides a high-efficiency and low-complexity approach for distributed resource allocation and ICI mitigation in the multicell MC DS-CDMA systems. Third, the DRA-2 scheme is capable of achieving a higher SE performance than the DRA-1 scheme, and the gain increases as the transmit SNR gets bigger. We can easily imply that this performance gain is achieved by using the OOP algorithm under the DRA-2 for ICI mitigation.

In Fig. 3(b), the MC DS-CDMA system with $N=1$ is reduced to the OFDMA system, where the CA is not employed. Apart from the similar observations of Fig. 3(a), we have the following observations obtained from Fig. 3(b). First, we observe that the SE performances for $M=8$ in Fig. 3(b) become worse than those in Fig. 3(a). This is because the multicarrier systems with employing DS spreading are able to provide another new degree-of-freedom for resource allocation. Second, seen from Fig. 3(b), the SE performance can be improved as the number of subcarriers $M$ increases, since a higher order of selecting diversity is achieved by the SA algorithms. Third, we can see that the performance of the RAIM scheme gets closer to that of the benchmark scheme when the OFDMA system employing more number of subcarriers. This observation implies the RAIM scheme is of high-efficiency, and it can be a promising candidate for large systems. In order to carry out further performance analysis, below we provide more comprehensive simulation results that can show how the SA, CA and ICI mitigation, respectively, affect the system performance.

For the sake of explicit comparison, Fig. 4 shows both the $\mathrm{SE}$ and bit error rate (BER) performance of the single-cell MC DS-CDMA systems employing the RAIM's distributed SA, i.e. the RAIM-SA algorithm and a range of existing SA algorithms. For the BER performance, we assume the binary phase shift keying (BPSK) baseband modulation. In Fig. 4, we observe that both the SE and BER performance achieved by the RAIM-SA algorithm can approach those of the optimum Hungarian algorithm. Furthermore, the RAIM-SA algorithm is capable of outperforming the worst case first (WCF) [23], while significantly outperforming the greedy and the worst subcarrier avoiding (WSA) algorithms [2] which are widely used in many references. The reason behind the above observations is that, the RAIM-SA is able to achieve a higher selection diversity than the other existing algorithms by assigning more users the best subchannels while avoiding allocating more users the worst subchannels. When the single-cell systems consider higher values of $M$ and $N$, the proposed RAIM-SA algorithm exploits higher selection diversity and can achieve larger performance gains over the other existing SA algorithms, which is the similar to the observations and conclusions in [23].

Fig. 5 compares the effect of ICI threshold $I_{t}$ on the SE performance of the systems employing different CA algorithms. Fig. 5 shows that, for each case, the best achievable SE of the RAIM-CA employing the optimum $I_{t}$ is close to that of the 


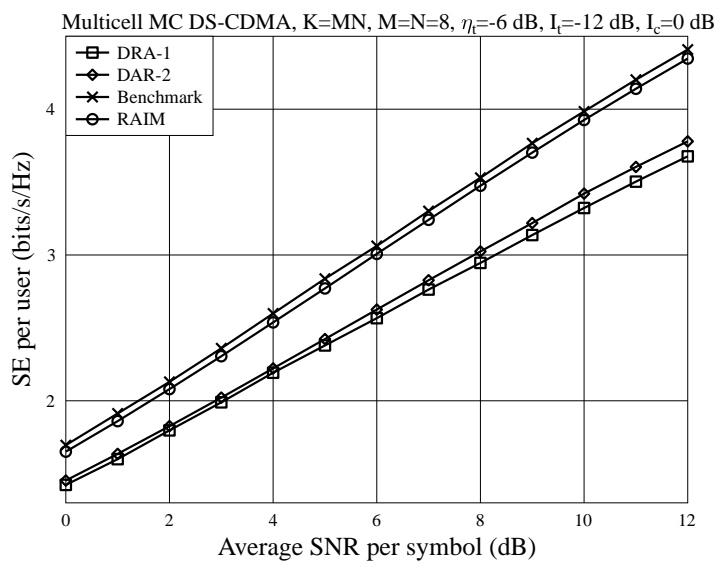

(a) $\mathrm{N}=\mathrm{M}$

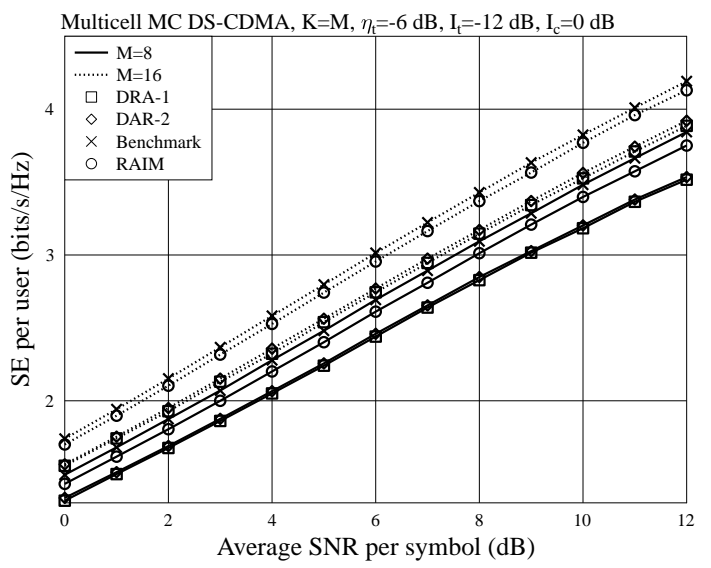

(b) $\mathrm{N}=1$

Fig. 3: SE of the multicell downlink MC DS-CDMA systems using various resource allocation and ICI mitigation schemes.

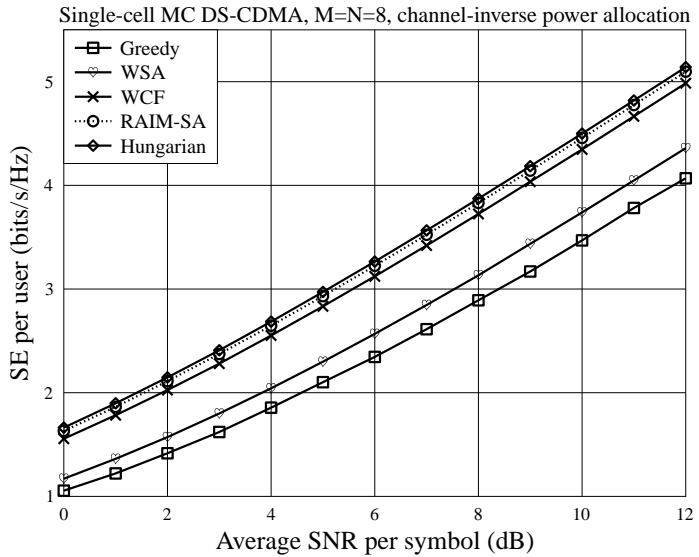

(a) SE

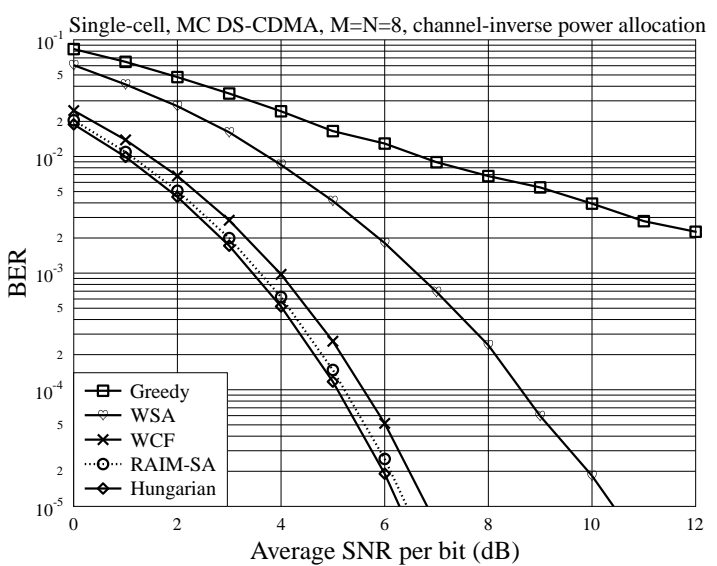

(b) BER

Fig. 4: Performance comparison of the various SA algorithms employed by the single-cell downlink MC DS-CDMA systems.

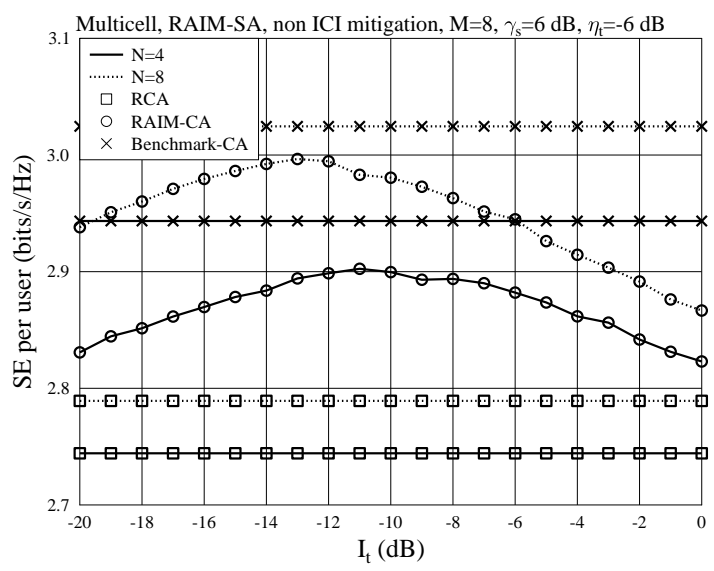

(a) SE per user of the system

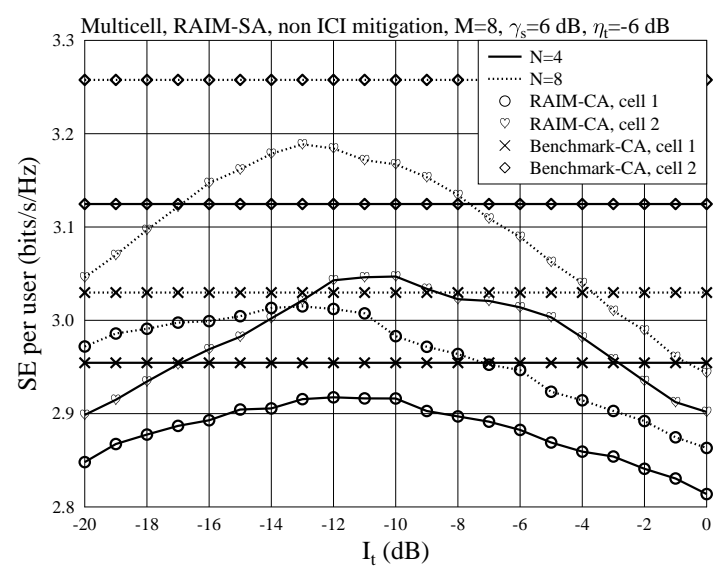

(b) SE per user of each cell

Fig. 5: SE of the multicell MC DS-CDMA systems employing the RAIM-SA and various CA algorithms but no ICI mitigation.

benchmark-CA, and is significantly higher than the SE achieved by the RCA. In Fig. 5(a), we see that, as $N$ increases from 4 to 8 , the highest SE achieved by the RAIM-CA gets closer to the SE of the Benchmark-CA, and the optimum $I_{t}$ range for the RAIM-CA shrinks and shifts from $-12 \mathrm{~dB} \leq I_{t} \leq-8 \mathrm{~dB}$ to $-14 \mathrm{~dB} \leq I_{t} \leq-12 \mathrm{~dB}$. This observation implies that, when 


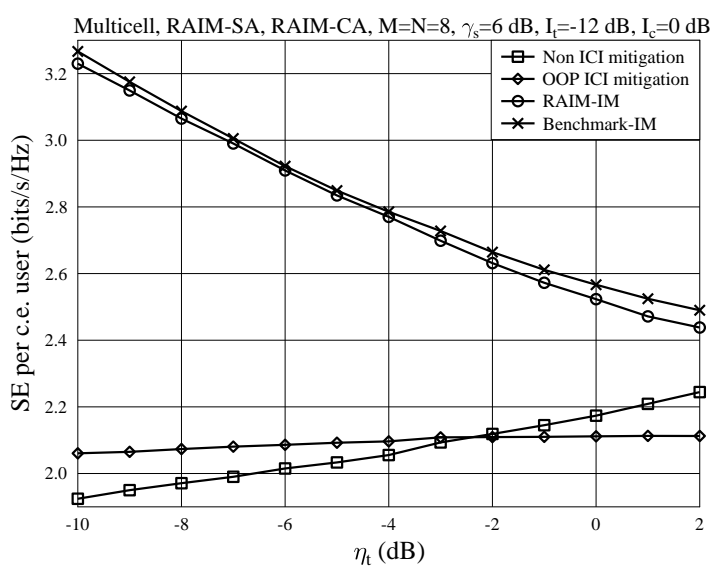

(a) SE per cell-edge user

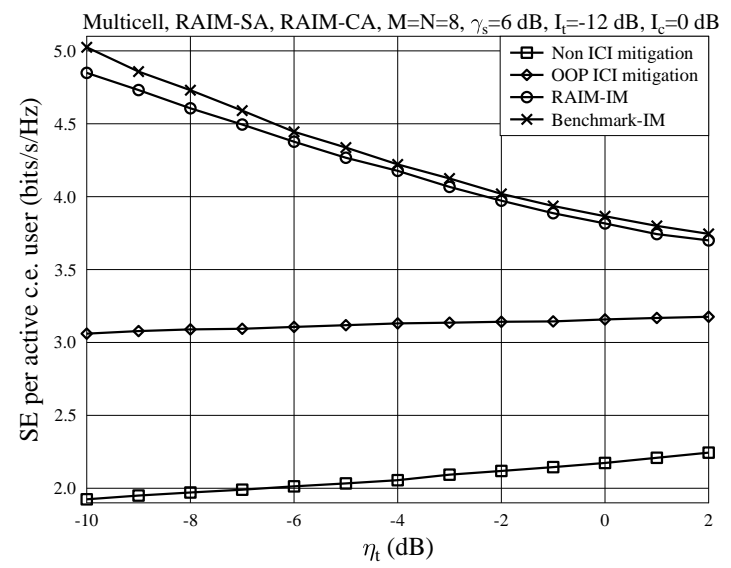

(b) SE per active cell-edge user

Fig. 6: SE of cell-edge users in the multicell downlink MC DS-CDMA systems employing various ICI mitigation algorithms, when different SIR thresholds are applied.

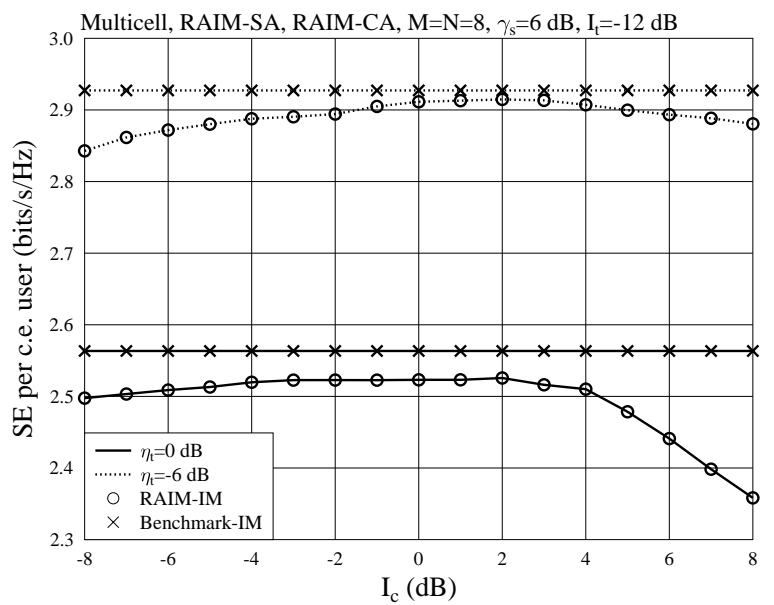

Fig. 7: SE of cell-edge users in the multicell downlink MC DSCDMA systems employing various ICI mitigation algorithms, when different ICI cooperation thresholds are applied.

the length of the spreading code increases, our RAIM-CA can achieve higher allocation diversity but it becomes more sensitive to the selection of the threshold $I_{t}$. For the sake of further evaluating the CA's performance, Fig. 5(b) shows the SE per user of one cell, which is obtained as the system's sum rate divided by the total number of users in the cell. We assume that all the CA algorithms considered in Fig. 5 are carried out successively by BSs 0,1 and then BS 2. Seen from Fig. 5(b), when employing the benchmark-CA or RAIM-CA algorithm, the SE performance for cell 2 is always better than that for cell 1 , and is certainly better than that for cell 0 which uses the RCA. Moreover, under the RAIM-CA, the SE performance for cell 1 changes more slowly than that for cell 2 as $I_{t}$ varies. This is because, the CA for the cell operated in the latter stage can make use of more ICI information in comparison to the CA for the cell operated in previous stages.

For the sake of explicit comparison, we address the SE performance of the ICI mitigation algorithms by focusing on the cell-edge users in the system. Fig. 6 shows the SE per cell- edge (c.e.) user and the SE per active cell-edge user, which are obtained by the sum rate of the cell-edge users divided by the total number of the cell-edge users and the number of the active cell-edge users, respectively. From Fig. 6, we observe that, the RAIM-IM algorithm always significantly outperforms the OOP algorithm and the case without ICI mitigation labeled as "non ICI mitigation", and attains nearly the similar performance as the Benchmark-IM algorithm. In Fig. 6(a), when $\eta_{t}$ increases, i.e. the number of cell-edge users get bigger, the SE performance achieved by the RAIM-IM and BenchmarkIM algorithms becomes worse. This is because that, at a given average SNR and an ICI cooperation threshold, the portion of the cell-edge users benefited by BS cooperation becomes smaller as $\eta_{t}$ increases. Note further that, more users could be turned off under the OOP algorithm when $\eta_{t}$ increases, hence the SE achieved by the OOP becomes lower than that obtained by doing nothing. From the above observations, we conclude that, it is very important for the ICI mitigation algorithms to choose proper SIR thresholds depending on various communication scenarios. In comparison with Fig. 6(a), Fig. 6(b) shows more SE performance gains obtained by the RAIM-IM over the OOP and non ICI mitigation algorithms. When focusing on the SE performance of the active cell-edge users, from Fig. 6(b) we also observe the OOP algorithm can significantly outperform the non ICI mitigation.

Fig. 7 shows the effect of the ICI cooperation threshold on the SE performance of the systems employing the RAIM-IM algorithm. In the figure, when $\eta_{t}$ increases from $-6 \mathrm{~dB}$ to $0 \mathrm{~dB}$, the optimum $I_{c}$ range required by the RAIM-IM achieving the highest $\mathrm{SE}$ is enlarged from $0 \mathrm{~dB} \leq I_{c} \leq 3 \mathrm{~dB}$ to $-3 \mathrm{~dB} \leq$ $I_{c} \leq 3 \mathrm{~dB}$. This observation implies that, the SE performance achieved by the RAIM-IM algorithm becomes less sensitive to the cooperation threshold $I_{c}$, as the SIR threshold increases. In general, when $I_{c}$ becomes smaller, the RAIM-IM algorithm tries to establish cooperation for more cell-edge users. By contrast, when it becomes larger, it allows cooperation for fewer cell-edge users. Hence, Fig. 7 implies that the threshold $I_{c}$ should be set to an appropriate value, so that a 'good' fraction of 
users experiencing strong ICI are identified for BS cooperation, in order to maximize the SE of cell-edge users. Moreover, from Figs. 6 and 7, we can conclude that the SIR thresholds $\eta_{t}$ for the RAIM-IM and the benchmark-IM algorithms should be chosen according to the design objectives, so as to yield a good tradeoff between performance and complexity.

\section{CONCLUSIONS}

In this paper, we have investigated the novel resource allocation and ICI mitigation in the multicell downlink MC DSCDMA systems. The analytical benchmark scheme for the multicell systems has been derived by solving and closely solving the mixed integer non-convex problems for maximizing the system's SE. Aiming to approach the performance of the benchmark scheme, we have proposed the fully distributed RAIM scheme with low implementation complexity and small backhaul resource required. Our simulation results have shown that, our RAIM scheme achieves the SE performance close to that of the benchmark scheme, and it can significantly outperform the other existing distributed schemes considered. Furthermore, the RAIM-SA algorithm requiring very little intracell channel knowledge can significantly outperform the other existing sub-optimum SA algorithms. Moreover, we have also shown that the RAIM-CA and RAIM-IM algorithms are very efficient and advantageous in terms of combating strong ICI. Therefore, the MC DS-CDMA associated with the RAIM scheme can constitute a promising candidate that facilitates practical implementation in future communication systems.

\section{APPENDIX}

In this appendix, we prove that the optimization problems in (25) and (27) are concave problems. We can observe that the constraints of (12), (13), (26) and (28) for the problems are all linear functions, hence we only need to prove the objective functions to be concave functions. Note that, each of the objective functions of (25) and (27) is in the same form, hence, in this section we only demonstrate the proof for one of the objective problems in (25). For notational simplicity, we drop some subindexes and scaling constants of all optimization variables in this section, so that the transformed objective functions in (25) with substituting $R_{k^{\prime}}^{\left(u^{\prime}\right)}$ by the transformed expression of (29) can be expressed as

$$
f=\sum_{k^{\prime} \in \mathcal{F}_{m}} R_{k^{\prime}}^{\left(u^{\prime}\right)}=\sum_{k^{\prime} \in \mathcal{F}_{m}} \sum_{n=0}^{N-1} c_{k^{\prime}, n} \log _{2}\left(1+\frac{1}{\sum_{n=0}^{N-1} c_{k^{\prime}, n}}\right) .
$$

Since all the users' rate expressions above are in the same form, we only need to prove that the rate function of user $k^{\prime}, R_{k^{\prime}}$, is a concave function. Let $\boldsymbol{H}\left(R_{k^{\prime}}\right)$ and $\lambda_{0}, \lambda_{1}, \ldots, \lambda_{N-1}$ be the Hessian matrix of function $R_{k^{\prime}}$ and the eigenvalues of $\boldsymbol{H}\left(R_{k^{\prime}}\right)$, respectively. In our multicell MC DS-CDMA systems, we may consider different lengths of the spreading codes, i.e. different values of $N \geq 2$.

Below, let us first consider the case when $N=2$. We readily know the Hessian matrix of function $\boldsymbol{H}\left(R_{k^{\prime}}\right)$ are given by

$$
\begin{aligned}
& \boldsymbol{H}\left(R_{k^{\prime}}\right)=\left[\begin{array}{ll}
H_{11} & H_{12} \\
H_{21} & H_{22}
\end{array}\right], \\
& H_{i j}=\frac{-1}{\ln 2\left(c_{k^{\prime}, 0}+c_{k^{\prime}, 1}+1\right)^{2}\left(c_{k^{\prime}, 0}+c_{k^{\prime}, 1}\right)}, \forall i, j
\end{aligned}
$$

Then, it finds that $\operatorname{tr}\left(\boldsymbol{H}\left(R_{k^{\prime}}\right)\right)=\lambda_{0}+\lambda_{1} \leq 0$. Furthermore, we can find that $\lambda_{0}=0$ and

$$
\begin{aligned}
\lambda_{2}= & \frac{-2}{\ln 2} \times\left[c_{k^{\prime}, 0}\left(c_{k^{\prime}, 0}^{2}+2 c_{k^{\prime}, 0}+1\right)+c_{k^{\prime}, 1}\left(c_{k^{\prime}, 1}^{2}+2 c_{k^{\prime}, 1}+1\right)\right. \\
& \left.+c_{k^{\prime}, 0} c_{k^{\prime}, 1}\left(3 c_{k^{\prime}, 0}+3 c_{k^{\prime}, 1}+4\right)\right]^{-1} .
\end{aligned}
$$

Hence, for the case $N=2, \boldsymbol{H}\left(R_{k^{\prime}}\right)$ is a negative semi-definite matrix and $R_{k^{\prime}}$ is concave, since $\lambda_{0}, \lambda_{1} \leq 0$.

Let us now consider the general case for $N \geq 3$. Due to the lack of space, in this paper we omit the derivation steps of the eigenvalues of $\boldsymbol{H}\left(R_{k^{\prime}}\right)$. According to our careful study, the eigenvalues can be given by

$$
\begin{aligned}
& \lambda_{n}= 0, n=0,1, \ldots, N-2, \\
& \lambda_{N-1}=-N \times\left(\phi_{1} \ln 2+\phi_{2} \ln 2+\phi_{3} \ln 2\right)^{-1} \\
& \phi_{1}=\sum_{n=0}^{N-1} c_{k^{\prime}, n}, \quad \phi_{2}=\sum_{n=0}^{N-1} 2 c_{k^{\prime}, n}^{2}+\sum_{n=0}^{N-1} \sum_{\tilde{n} \neq n, \tilde{n}=0}^{N-1} c_{k^{\prime}, n} c_{k^{\prime}, \tilde{n}} \\
& \phi_{3}=\sum_{n=0}^{N-1} 2 c_{k^{\prime}, n}^{3}+\sum_{n=0}^{N-1} \sum_{\tilde{n} \neq n, \tilde{n}=0}^{N-1} 3 c_{k^{\prime}, n}^{2} c_{k^{\prime}, \tilde{n}} \\
& \quad+\sum_{n=0}^{N-1} \sum_{\tilde{n} \neq n, \tilde{n}=0}^{N-1} \sum_{\hat{n} \neq \tilde{n} \neq n, \hat{n}=0}^{N-1} 6 c_{k^{\prime}, n} c_{k^{\prime}, \tilde{n}} c_{k^{\prime}, \hat{n}}
\end{aligned}
$$

We find that all the eigenvalues are smaller or equal to zero, i.e. $\lambda_{0}, \lambda_{1}, \ldots, \lambda_{N-1} \leq 0$. Therefore, we can conclude that the rate function $R_{k^{\prime}}$ is concave, and the objective functions in (25) and (27) are joint concave functions with respect to (w.r.t.) the CA indicators $\left\{c_{k^{\prime}, n}, \forall k^{\prime}, n\right\}$ and $\left\{c_{k^{\prime \prime}, n}, \forall k^{\prime \prime}, n\right\}$, respectively.

\section{REFERENCES}

[1] K. Kim and J. Kim, "A 2-D subcarrier allocation scheme for capacity enhancement in a clustered OFDM system," IEICE Trans. on Commun., vol. E90-B, pp. 1880 - 1883, July 2007.

[2] T. Liu, C. Yang, and L. L. Yang, "A low-complexity subcarrier-power allocation scheme for frequency-division multiple-access systems," IEEE Trans. Wireless Commun., vol. 9, no. 5, pp. 1564 - 1570, May 2010.

[3] M. G. Kibria and L. Shan, "Resource allocation optimization for users with different levels of service in multicarrier systems," IEEE Signal Processing Letters, vol. 22, no. 11, pp. 1869-1873, 2015.

[4] V. Tervo, A. Tlli, and T. Matsumoto, "PAPR constrained power allocation for multicarrier transmission in multiuser SIMO communications," IEEE Trans. Wireless Commun., vol. 15, no. 4, pp. 2458-2473, 2016.

[5] L. Venturino, A. Zappone, C. Risi, and S. Buzzi, "Energy-efficient scheduling and power allocation in downlink OFDMA networks with base station coordination," IEEE Trans. Wireless Commun., vol. 14, no. 1, pp. 1-14, Jan. 2015.

[6] E. Baktash, M. Rasti, and E. Hossain, "Resource allocation for dynamic intra-cell subcarrier reuse in cooperative OFDMA wireless networks," IEEE Trans. Mobile Computing, vol. 14, no. 7, pp. 1475-1489, July 2015.

[7] M. Fathi and E. Karipidis, "Distributed resource optimization in multicell OFDMA networks," Proc. of IEEE WCNC), pp. 1316 - 1320, April 2012.

[8] K. W. Choi, E. Hossain, and D. I. Kim, "Downlink subchannel and power allocation in multi-cell OFDMA cognitive radio networks," IEEE Trans. Wireless Commun., vol. 10, no. 7, pp. 2259-2271, July 2011.

[9] B. Ozbek, D. L. Ruyet, and M. Pischella "Adaptive reduced feedback links for distributed power allocation in multicell MISO-OFDMA networks," IEEE Wireless Commun. Letters, vol. 3, no. 2, pp. 141-144, April 2014.

[10] Y. Yu, E. Dutkiewicz, X. Huang, and M. Mueck, "Downlink resource allocation for next generation wireless networks with inter-cell interference," IEEE Trans. Wireless Commun., vol. 12, no. 4, pp. 1783-1793, April 2013.

[11] D. W. K. Ng and R. Schober, "Resource allocation and scheduling in multi-cell OFDMA systems with decode-and-forward relaying," IEEE Trans. Wireless Commun., vol. 10, no. 7, pp. 2246 - 2258, July 2011. 
[12] H. Zhang, L. Venturino, N. Prasad, P. Li, S. Rangarajan, and X. Wang, "Weighted sum-rate maximization in multi-cell networks via coordinated scheduling and discrete power control," IEEE Journal on Selected Areas in Communications, vol. 29, no. 6, pp. 1214-1224, June 2011.

[13] H. Galeana-Zapien and R. Ferrus, "Design and evaluation of a backhaulaware base station assignment algorithm for OFDMA-based cellular networks," IEEE Trans. Wireless Commun., vol. 9, no. 10, pp. 3226-3237, Oct. 2010

[14] D. W. K. Ng, E. S. Lo, and R. Schober, "Energy-efficient resource allocation in multi-cell OFDMA systems with limited backhaul capacity," IEEE Trans. Wireless Commun., vol. 11, no. 10, pp. 3618 - 3631, Oct. 2012.

[15] L. L. Yang and L. Hanzo, "Multicarrier DS-CDMA: a multiple access scheme for ubiquitous broadband wireless communications," IEEE Communications Magazine, vol. 41, no. 10, pp. 116 - 124, Oct. 2003.

[16] L. L. Yang, Multicarrier Communications. John Wiley, 2009.

[17] L. Loyola and T. Miki, "Speed-aware multicarrier transmission and resource allocation scheme for the uplink channel of highly mobile networks," in Proceedings of IEEE Topical Conference on Wireless Communication Technology., Oct. 2003, pp. 117-118.

[18] C.-W. Chang and L.-C. Wang, "WLC03-3: A joint subcarrier power allocation and interference avoidance code assignment strategy for multirate MC-DS-CDMA with TF-domain spreading," in Proc. of IEEE GLOBECOM, Nov. 2006, pp. 1-5.

[19] C.-W. Chang and C.-C. Kuo, "A low interference time-slicing code assignment for the 2D-Spread MC-DS-CDMA systems," in Proc. of IEEE VTC Spring, April 2009, pp. 1-5.

[20] _ "A novel interference-avoidance code reassignment for downlink two-dimensional-spread MC-DS-CDMA systems with power control," IEEE Trans. Vehi. Tech., vol. 59, no. 6, pp. 3104-3108, July 2010

[21] Z. Wang, D. Yang, and L. Milstein, "Multi-user resource allocation for a distributed multi-carrier DS-CDMA network," IEEE Trans. Commun., vol. 60, no. 1, pp. 143-152, 2012.

[22] W. J. Huang, W. W. Hu, C. P. Li, and J. C. Chen, "Novel metric-based PAPR reduction schemes for MC-CDMA systems," IEEE Trans. Vehi. Tech., vol. 64, no. 9, pp. 3982-3989, 2015.

[23] J. Shi and L. L. Yang, "Novel subcarrier-allocation schemes for downlink MC DS-CDMA systems," IEEE Trans. Wireless Commun., vol. 13, no. 10 , pp. 5716-5728, Oct. 2014.

[24] C. Y. Ho and C.-Y. Huang, "Non-cooperative multi-cell resource allocation and modulation adaptation for maximizing energy efficiency in uplink OFDMA cellular networks," IEEE Wireless Commun. Letters, vol. 1, no. 5, pp. 420-423, 2012.

[25] S.-Y. Kim, J.-A. Kwon, and J.-W. Lee, "Sum-rate maximization for multicell OFDMA systems," IEEE Transactions on Vehicular Technology, vol. 64, no. 9, pp. 4158-4169, 2015.

[26] S. H. Ali and V. C. M. Leung, "Dynamic frequency allocation in fractional frequency reused OFDMA networks," IEEE Trans. Wireless Commun., vol. 8, no. 8, pp. 4286 - 4295, Aug. 2009.

[27] A. J. Viterbi, A. M. Viterbi, and E. Zehavi, "Other-cell interference in cellular power-controlled CDMA," IEEE Trans. Commun., vol. 42, no. 234, pp. 1501 - 1504, Feb. 1994.

[28] D. Gesbert, S. G. Kiani, A. Gjendemsjo, and G. E. Oien, "Adaptation, coordination, and distributed resource allocation in interference-limited wireless networks," Proceedings of the IEEE, vol. 95, no. 12, pp. 23932409, Dec. 2007.

[29] S. Alamouti, "A simple transmit diversity technique for wireless communications," IEEE Journal on Selected Areas in Communications, vol. 16, no. 8, pp. 1451 - 1458, Oct. 1998.

[30] J. Shi and L. L. Yang, "Bidirectional worst subchannel avoiding versus best subchannel seeking subcarrier-allocation in downlink OFDMA systems," IEEE Trans. Vehi. Tech., Early Access, 2015.

[31] O. Nwamadi, X. Zhu, and A. Nandi, "Dynamic subcarrier allocation for single carrier - FDMA systems," in Proc. of EUSIPCO, Aug. 2008.

[32] S. boyd and L. Vandenberghe, Convex Optimization. Cambridge, UK: Cambridge University Press, 2004.

[33] S. G. Kiani, G. E. Oien, and D. Gesbert, "Maximizing multicell capacity using distributed power allocation and scheduling," Proc. of IEEE WCNC, pp. 1690-1694, March 2007

[34] H. W. Kuhn, "The Hungarian method for the assignment problem," Naval Research Logistics Quarterly, vol. 2, pp. 83 - 97, 1995.

[35] M. S. K. Lau, S. P. Yue, K. V. Ling, and J. M. Maciejowski, "A comparison of interior point and active set methods for FPGA implementation of model predictive control," in European Control Conference (ECC), Aug 2009, pp. $156-161$

[36] N. K. Karmarkar, "A new polynomial-time algorithm for linear programming," Combinatorica 4, pp. 373 - 395, 1984.
[37] I. J. Lustig, R. E. Marsten, and D. F. Shanno, "Feature article-interior point methods for linear programming: Computational state of the art," ORSA Journal on Computing, vol. 6, no. 1, pp. 1 - 14, 1994.

[38] A. Goldsmith, Wireless Communications. U.K.: Cambridge University Press, 2005. 